\title{
Superconductivity in carbon nanotubes
}

\author{
Junji Haruyama1-3 \\ ${ }^{1}$ Aoyama Gakuin University, 5-10-1 Fuchinobe, Sagamihara, Kanagawa 229-8558, Japan \\ 2Institute for Solid State Physics, University of Tokyo, Kashiwanoha 5-1-5, Kashiwa, Chiba \\ 277-8581, Japan \\ 3 Japan Science and Technology Agency-CREST, 4-1-8 Hon-machi, Kawaguchi, Saitama \\ 332-0012, Japan
}

\begin{abstract}
Carbon nanotubes (CNTs), which are one-dimensional (1D) molecular conductors, have attracted considerable attention from viewpoints of the novel structures, electronic states, quantum phenomena, and also applications to new opt and electronic devices. However, mostly none reported on superconductivity (SC) and its correlation with 1D electron phenomena. In the present study, I report on SC with the world-highest transition temperature $\left(\mathrm{T}_{\mathrm{c}}\right)$ of $12 \mathrm{~K}(1)$ in arrays of multi-walled CNTs (MWNTs) with entirely end-bonded electrode junction and (2) in highly uniform thin films of boron-doped single-walled CNTs (B-SWNTs). In the former one, I reveal correlation of SC with Tomonaga-Luttinger liquid (TLL) state, which is a collective phenomenon arising from repulsive Coulomb interaction between electrons confined in 1D ballistic charge transport regimes. In the latter one, I clarify correlation of SC with van Hove singularities (VHS) in electronic density of states (DOS) in the SWNT. Based on this thin film, I show fabrication of paper-like thin films consisting of pseudo two-dimensional network of weakly coupled $B$-SWNTs (the so-called Buckypaper) and show an enhancement of the onset $T_{c}$ up to $19 \mathrm{~K}$ by applying only a small pressure. In section 4 , I discuss about possibility for realization of quantum spin entangler and quantum bit utilizing a hybrid system of CNTs/superconductor, which is associated with the section 1. Because CNTs have strong spin coherence and possibly strong spin entanglement in spin singlet (Cooper pair), it must allow realization of quantum spin entangler and bit without decoherence problem in near future. The present observations will shed light to feasibility of CNTs as a 1D superconductor and also research of $1 \mathrm{D}$ electron correlation. They promise application to novel quantum devices.
\end{abstract}




\section{Superconductivity in multi-walled carbon nanotubes: Interplay of Tomonaga -Luttinger liquid and superconductive phase}

It is well known that the phonon- mediated attractive electron-electron interaction leads to a superconductive instability in $2 \mathrm{D}$ and $3 \mathrm{D}$ conductors. In particular, high-Tc SC favors 2D space such as cupper-oxide high- $\mathrm{T}_{\mathrm{c}}$ superconductors. Here, how does SC like 1D space? In CNTs, which is one of typical 1D conductors, a variety of 1D quantum phenomena have been reported. However, intrinsic SC has been reported in the CNTs only by a few groups [1-3]. Nevertheless, the SC in a CNT is attracting considerable attention for the following reasons: (1) The curvature resulting from a small diameter $(<<\sim 1 \mathrm{~nm})$ can bring high a $\mathrm{T}_{\mathrm{c}}$ due to a strong coupling of $\sigma-\pi$ band electrons, which arises from formation of $\mathrm{sp}^{3}$ hybrid orbitals and $\sigma-\pi$ mix band, with radial breathing phonon mode. (2) The perfect alignment of the Fermi level $\left(\mathrm{E}_{\mathrm{F}}\right)$ to a van Hove singularity (VHS) can also lead to high $\mathrm{T}_{\mathrm{c}}$ due to the appearance of an extremely large electronic density of states (DOS). (3) It can provide an insight into the 1D electron correlation. Thus, study of SC in CNTs can shed lights to feasibility of $1 \mathrm{D}$ conductors as superconductors.

In contrast, many quantum phenomena and electronic states tend to obstruct appearance of SC in 1D space. For instance, Coulomb repulsion in TLLs, energy gaps arising from Pierls transition, very small electronic DOS in energy regimes out of VHSs, and phase fluctuation can such obstructions. In particularly, TLL is one of typical 1D quantum phenomena. The TLL provides the relevant theoretical description [4-6], representing a collective electronic state (e.g., showing spin-charge separation) that arises from the repulsive Coulomb interaction between electrons confined in a 1D ballistic conductance regime. Many previous works have reported TLL features in CNT systems. It is known that the tunneling density of states $v(E)$ for the TLLs decreases following a power-law dependence on energy, $v(E) \propto E^{\alpha}$, where the power $\alpha$ is given by different expressions according to the different tunneling regimes $[4,5,12]$. Most previous works on CNTs have been dealing with the regime of strong Coulomb interaction.

Here, SC is a phenomenon arising from attractive Coulomb interaction via phonon, which is in contradiction to the Coulomb repulsion leading to the TLL state. This leads naturally to the question of how the SC can develop in the CNTs, with the consequent transition from the TLL $[7-11,14]$. The interplay between the TLL state and intrinsic SC has been only reported in our MWNTs with a significant number $N$ of shells participating in the conduction [2]. The results were qualitatively consistent with low-energy theories of CNTs [8], which had already predicted the breakdown of the TLL at low temperatures in nanotube bundles with a strong intertube electrostatic coupling between the different $1 \mathrm{D}$ conduction channels.

In the MWNTs, however, the interplay of the SC with the TLL and the value of $\mathrm{T}_{\mathrm{c}}$ as high as $12 \mathrm{~K}$ still had to be quantitatively understood, as the samples were not intentionally doped and the number of $1 \mathrm{D}$ conduction channels was unknown. On the other hand, the high $\mathrm{T}_{\mathrm{c}}$ value was in approximate agreement with $\mathrm{T}_{\mathrm{c}}$ 's $=\sim 10 \mathrm{~K}$ of $\mathrm{CaC}_{6}$ and boron $(B)$-doped diamond. Indeed, low-energy theories of MWNTs have suggested the importance of having a large number of 1D conduction channels for the development of a SC instability [10]. Therefore, it is crucial to clarify the correlation between the appearance of the SC phase and carrier doping in MWNTs. $B$-doping has been actually successfully achieved following known methods $[15,16]$. 
In this section, I report on SC in array of the MWNTs and reveals correlation with TLL state. I find an abrupt resistance drop due to $\mathrm{SC}$ at a $\mathrm{T}_{\mathrm{c}}$ as high as $12 \mathrm{~K}$ in the entirely end-bonded MWNTs. In contrast, the partially end-bonded MWNTs show very small resistance drop at low temperatures. It reveals that a gradual transition from TLL behavior to a SC regime can start only from low $e V / k T$ values at low temperatures, but the development of the SC enhances the regime to high $e V / k T$ region. Phase diagram obtained from low-energy theory reveals that such a transition is actually possible. Nuclear Magnetic Resonance (NMR) measurements ascertain the presence of substitutional boron doping for SC in the MWNTs.

At first, I report on the possibility of unintentional $B$-doping in our MWNTs, which were synthesized by chemical vapor deposition using Fe/Co catalyst and methanol gas in the nanopores of the alumina template $[2,19]$. Boron has been intentionally used only for the enhancement of the chemical reaction of the Fe/Co catalyst $[2,19]$. Figure 1 shows results of nuclear magnetic resonance (NMR) measurements of our MWNTs with three different boron concentrations $\left(N_{B}\right)$ in the catalyst. Evident peaks can be observed at $0 \sim 5 \mathrm{ppm}$ in individual samples. The peak position implies the presence of chemical bonds of $B-C$ and, hence, substitutional $B$ doping in the carbon network of the MWNTs. Moreover, at least five-times higher intensity of NMR signal peak for the $B$ - $C$ was detected in the $B$-MWNTs with $N_{B}=\sim 2$ at.\%, compared with other $B$-MWNTs with different $N_{B}$ values. Very importantly, we find that only the $B$-MWNTs with $N_{B}=\sim 2$ at. \% showed SC with high $\mathrm{T}_{\mathrm{c}}$ (Fig. $3(\mathrm{a})$ ) and also present signature of SC (Fig. 3(b)) with the highest reproducibility. This result implies that the most suitable condition for substitutional $B$ doping into the MWNTs, which leads to appearance of the SC, is $\sim 2$ at.\%. This is consistent with our recent discovery [20] and a theory [21]. The small $B$ concentration allows the CNTs remaining a 1D ballistic charge transport regime.

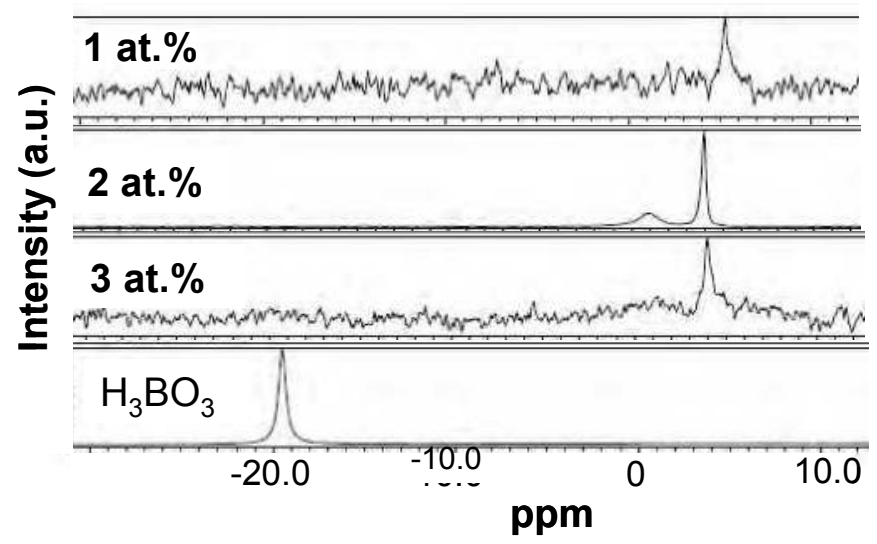

Fig. 1. NMR measurement results of the MWNTs that were synthesized from Fe/Co with different $B$ concentration noted by atomic \%, using ${ }^{11} \mathrm{C}$. Result of $\mathrm{H}_{3} \mathrm{BO}_{3}$ is a reference. $\mathrm{H}_{3} \mathrm{BO}_{3}$ was mixed in $\mathrm{FeSO}_{4}$ and $\mathrm{CoSO}_{4}$, and then $\mathrm{Fe} / \mathrm{Co}$ catalyst including $B$ was electrochemically deposited into the bottom ends of nanopores of alumina template $[5,19]$. 


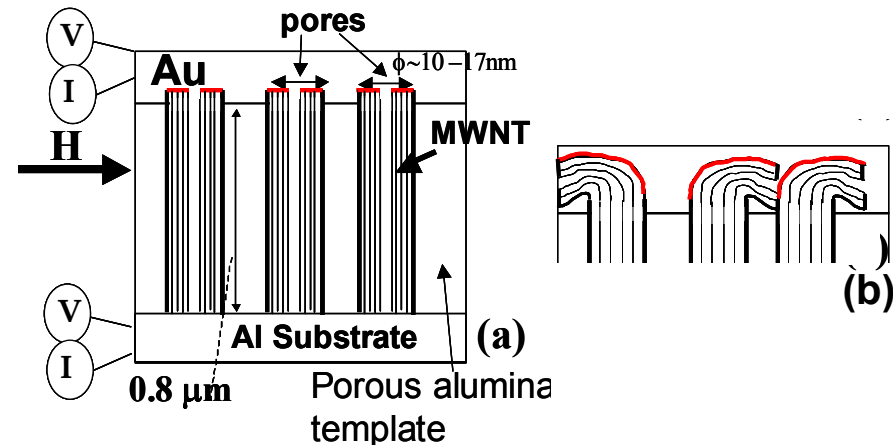

Fig. 2. Schematic cross sections of array of MWNTs with (a) entirely end-bonded and (b) partially end-bonded junctions between Au electrode and top ends of MWNTs. The red lines indicate the interfaces between the MWNTs and the electrodes.

Figure 2 shows the schematic cross sections of our samples, which include arrays of $B$-MWNTs with (a) entirely end-bonded $(N=9)$ and $(b)$ partially end-bonded $(1<N<9)$ junctions to $\mathrm{Au}$ electrodes [2]. They were fabricated by entirely and partially cutting the excess top portions of MWNTs, which were grown up from the nanopores and accumulated on alumina membrane, respectively. No cutting resulted in bulk junction, which has electrical contact to only the outermost shell (i.e., $N=1$ ).
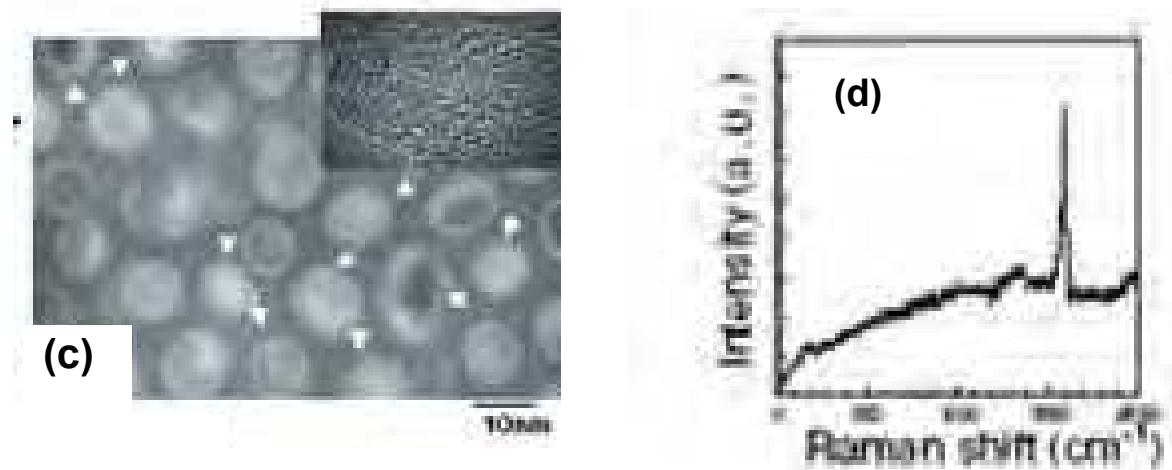

Fig. 2. (c) Plane TEM image of an array of MWNTs. Inset: High resolution TEM image of one MWNT. (d) Resonant Raman spectrum of the MWNT. The significantly sharp peak indicates G band.

Figure 2(c) shows a planer TEM image of the sample [2]. One can confirm presence of MWNTs in most pores. Figure 2(d) shows measurement result of resonant Raman spectrum. Peak height of $G$ band is significantly greater than that of $D$ band. This strongly indicates high quality of the MWNTs. 

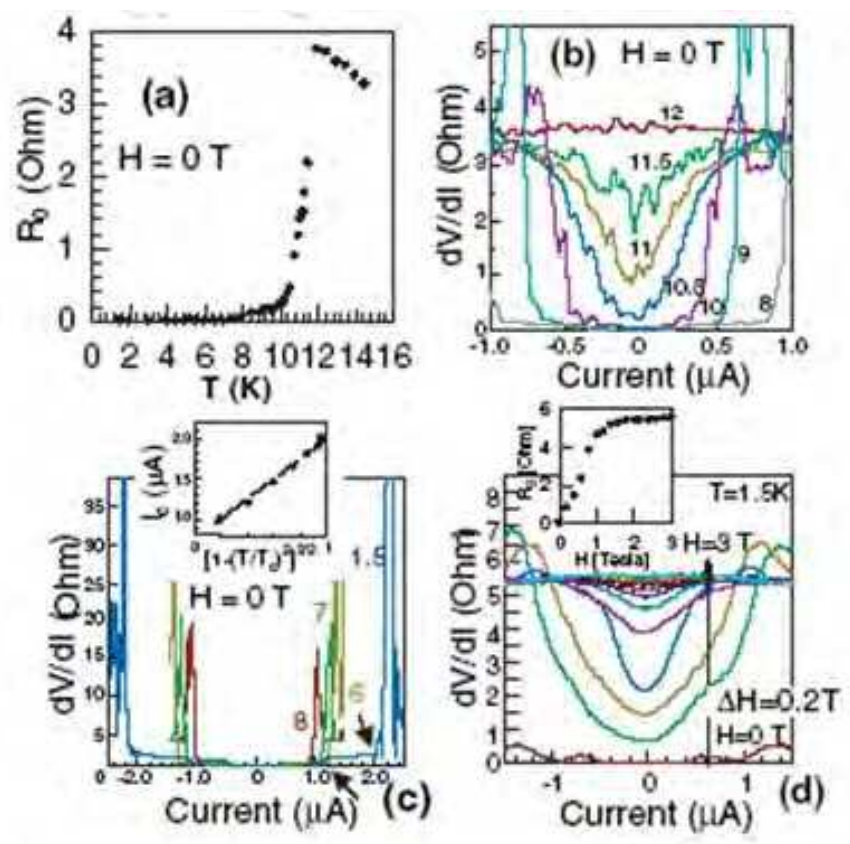

Fig. 3. (a) Superconductivity with $\mathrm{T}_{\mathrm{c}}=12 \mathrm{~K}$ in the sample with junction type (a) (b) Differential resistance vs. Temperature (Kelvin) for (a) $(\mathrm{T}>8 \mathrm{~K})$. (c) that for $\mathrm{T}<8 \mathrm{~K}$ Inset : Critical current vs. Normalized temperature for GL theory (d) Differential resistance vs. magnetic fields applied perpendicular to tube axis

Figure 3 shows superconducting behaviors of the entirely end-bonded MWNTs as shown in Fig. 2(a). In Fig. 3(a), zero-bias resistance $\left(R_{0}\right)$ abruptly starts to drop from $T_{c}=12 \mathrm{~K}$ and attains to zero-ohm at $\mathrm{T}=7.8 \mathrm{~K}$, when residual resistance, which arises from contact resistance, was subtracted. Figure $3(\mathrm{~b})$ shows the differential resistance dips for various temperatures $>8 \mathrm{~K}$. As temperature decreases, the dip monotonically deepens and mostly attains to zero-ohm at the center point at $\mathrm{T}=8 \mathrm{~K}$. This is consistent with Fig. 3(a). Figure 3(c) shows differential resistance at $\mathrm{T}<8 \mathrm{~K}$. When current increases at each temperature, zero differential resistance dip suddenly disappears at critical current $\left(I_{c}\right)$. The inset shows relationship of $I_{c}$ vs. temperature normalized for GL equation. It exhibits a linear relationship and turns out that Figs.3(a) - (c) are attributed to SC. Figure 3(d) shows differential resistance dips as a function of magnetic field. The dip rapidly disappears as the field increases. 


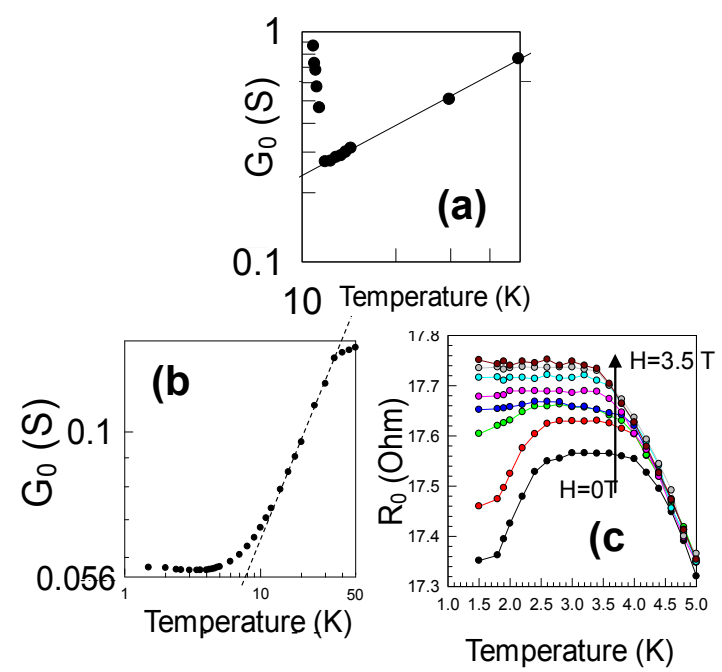

Fig. 4. Doubly logarithmic scales of zero-bias conductance $G_{0}$ and temperature for (a) entire and (b) partial end junctions. (c) Magnetic field dependence of zero-bias resistance $R_{0}$ as a function of the temperature, corresponding to (b).

Figure 4 shows the doubly logarithmic scales of zero-bias conductance $\mathrm{G}_{0}$ and temperature for the two different junctions shown in Fig. 2. The observed power law behaviors in the different types of junctions $\left(\mathrm{G}_{0} \propto \mathrm{T}^{\alpha} ; \alpha=0.7\right.$ and 0.8 in (a) and (b) respectively) are in excellent agreement with the previous reports of TLL behavior in CNTs [2, 5]. As shown in Fig. 4(a), the abrupt $\mathrm{G}_{0}$ increase (i.e., $\mathrm{SC}$ regime) appears overcoming the TLL behavior (i.e., the power law at $\mathrm{T}>12 \mathrm{~K}$ ) at $\mathrm{T}_{\mathrm{c}}=12 \mathrm{~K}$ in the entirely end-bonded $B$-MWNTs. The origin of this effect was in the large screening of the Coulomb interaction, arising from the electrostatic coupling between a large number of $1 \mathrm{D}$ conduction channels due to boron doping and also the large $N$ value originating from entire end-bonding of the $B$-MWNTs $[2,8]$.

In contrast, in the partially end-bonded $B$-MWNTs, only a slight and gradual $\mathrm{G}_{0}$ increase at $\mathrm{T}<2.5 \mathrm{~K}$ (i.e., a signature of SC correlations) can be observed as shown in Fig. 4 (b). As we implied in ref. [5], the appearance of SC and the correlations of power laws with $\mathrm{G}_{0}$ increase were strongly correlated with the values of $N$ as shown in Fig. 3. Because we could confirm that the abrupt $\mathrm{G}_{0}$ increase in Fig. 4(a) was attributed to SC as mentioned above [2], it can be concluded that the small $\mathrm{G}_{0}$ increase observed in Fig. 4(b) is also attributed to SC. The gradual $\mathrm{G}_{0}$ increase corresponds to the slight and gradual resistance drop shown in Fig. 4(c). With an increase in the magnetic field, the drop in $\mathrm{R}_{0}$ disappears rapidly. This behavior actually agrees with that of conventional superconductors.

In Fig. 4(b), the power law is observable up to $\mathrm{T}=\sim 40 \mathrm{~K}$ for $\alpha=\sim 0.7$, while it gradually starts to deviate below $\mathrm{T}=\sim 12 \mathrm{~K}$. At $\mathrm{T}<\sim 6 \mathrm{~K}$, it saturates completely. Subsequently, a small increase in $\mathrm{G}_{0}$ due to the SC correlations appears at $\mathrm{T}<\sim 2.5 \mathrm{~K}$. However, the trend towards the SC transition is not completed above $\mathrm{T}=1.5 \mathrm{~K}$ in this case. This is consistent with the fact that the SC phase requires the coherent transport of Cooper pairs along the directions transverse to the CNTs, which cannot be established when only part of the shells are 
electrically active and the number of conductance channels is smaller as in the partially end-bonded $B$-MWNTs with the small $N$ values $[2,8]$. This also implies that the Coulomb interaction cannot be sufficiently screened to allow for the development of SC correlations. In fact, $B$-MWNTs with $N=1$ exhibited no $\mathrm{G}_{0}$ increase, consequent with the absence of screening of the Coulomb interaction.
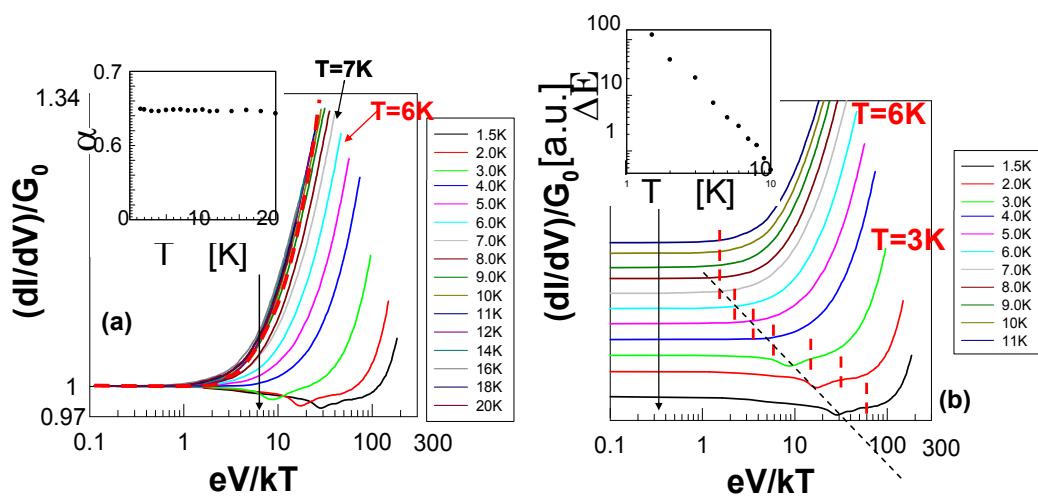

Fig. 5. (a) Doubly logarithmic scales on normalized differential condūctance vs. $e V / k T$, corresponding to TLL formula in $\mathrm{eV} \gg k T$ regime [13]. The red dotted line gives the best fitting result by eq.(1). Inset: power value $\alpha$ vs. temperature estimated from main panel. (b) Transform of $(d I / d V) / \mathrm{G}_{0}$ in Fig. 5(a) to arbitrary units. The long black-dotted line connects the right edges of the conductance peaks. Inset: $\Delta \mathrm{E}_{\mathrm{c}}$ vs. temperature on doubly logarithmic scales. $\Delta \mathrm{E}_{\mathrm{c}}=\mathrm{E}_{\mathrm{c}}(\mathrm{T})-$ $E_{c}(T=7 K)$, where $E_{c}(T)$ is the boundary point between the saturation and power law regions at each temperature, as shown by the short red-dotted lines in the main panel.

On the other hand, besides the dependence on temperature, the power law behavior of the observables as a function of the bias voltage $V$ provides absolute evidence of the TLL behavior $[4,5,13]$. Thus, it is crucial to investigate the regime $e V>>k T$ to discuss in depth the transition from TLL behavior to the SC phase. In this sense, Fig. 5(a) exhibits doubly logarithmic plots of the normalized differential conductance vs. $e V / k T$, which is one of the typical dependences to check the TLL behavior for $e V>>k T$ [13]. It shows that the entire data collapse suitably onto a single universal curve at $\mathrm{T} \geq \sim 7 \mathrm{~K}$. This agrees with the results reported in previous investigations of TLL behavior $[4,5,13]$. As shown by the red dotted line, this universal curve can be fitted by the following result for a TLL [13]

$$
\frac{G(V, T)}{G(V=0, T)}=\cosh \left(\gamma \frac{e V}{2 k T}\right) \frac{1}{\left|\Gamma\left(\frac{1+\alpha}{2}\right)\right|^{2}} \times\left|\Gamma\left(\frac{1+\alpha}{2}+\gamma \frac{i e V}{2 \pi k T}\right)\right|^{2}
$$


where $V$ is the applied voltage; $\Gamma(\mathrm{x})$, the Gamma function; $k$, the Boltzmann constant; and $\gamma$, the inverse number of the measured junctions $M$ weighted by their resistance [13]. For $M$ junctions in series, the value of $\gamma$ should be within $\gamma=1 / M$. The best fit gives the parameter $\gamma$ $=\sim 0.7$, which is mostly in good agreement with $\gamma=1 / M=0.5$ for $M=2$ of our individual MWNTs with two end junctions. Therefore, we conclude that the universal curve can be actually attributed to TLL behavior at $\mathrm{T} \geq \sim 7 \mathrm{~K}[2]$.

However, we importantly find appearance of a deviation from the universal curve in the low temperature regime (e.g., at $\mathrm{T} \leq 6 \mathrm{~K}$ ) in Fig. 5. As shown by red lines in Fig. 5(b), this deviation originates from a monotonic increase in the width of the low-eV/kT saturation region (i.e., $e V / k T<\sim 2$ at $\mathrm{T}=6 \mathrm{~K}$ ) in the universal curve at $\mathrm{T} \leq \sim 6 \mathrm{~K}$. The inset of Fig. $5(\mathrm{~b})$ shows $\Delta \mathrm{E}_{\mathrm{c}}$ (width of the saturation region) as a function of temperature in a doubly logarithmic scale. Indeed, we can confirm that $\Delta \mathrm{E}_{\mathrm{c}}$ actually increases with a decrease in temperature, following power law dependence.

Quite remarkably, it should be noted that the value of the critical temperature of $6 \mathrm{~K}$ for the deviation of the universal curve is in excellent agreement with that of the saturation region in Fig. 4(b). This agreement strongly suggests that the deviation at $\mathrm{T} \leq \sim 6 \mathrm{~K}$ in Fig. 4 for $\mathrm{eV}$ $>>k T$ is associated with the appearance of the SC regime. Figure $5(\mathrm{~b})$ supports this argument, because it suggests that the increase in the width of the saturation region is caused by the appearance (insertion) of the conductance peak due to emergence of the SC regime. In particular, this is evident at $\mathrm{T} \leq 3 \mathrm{~K}$, where $\mathrm{G}_{0}$ is seen to increase in Fig. 4(b) [22]. This implies that the emergence of the $\mathrm{SC}$ regime at low $e V / k T$ leads to the deviation from the TLL states [17].

In the conventional theory of a TLL, the width of the low-eV/kT saturation region $(<\sim 1)$ reflects the regime of $e V / k T<\Delta \mathrm{E}=h v_{F} / L$, where $\Delta \mathrm{E}$ is the energy spacing of the quantized electronic orbital formed in the CNTs in the ballistic charge transport regime [18]. This means that the noise from thermal fluctuations smears the effects of the bias voltage (i.e., power laws) out in the low-eV/kT regime $(<\sim 1)$. In contrast, one should notice that insertion of the conductance peak in Fig. 5(b) enhances this thermal smearing region to high-eV/kT regime $(\sim 100)$ at $\mathrm{T}=1.5 \mathrm{~K}$. This is very anomalous and, hence, strongly implies a possibility that appearance of the SC regime introduces superconducting gap $\Delta_{\mathrm{SC}}$ in the system, resulting in $\Delta \mathrm{E}+\Delta_{\mathrm{SC}}$.

Moreover, the inset of Fig. 5(a) shows the values of $\alpha$, which were estimated from the main panel of Fig. 5(a), as a function of temperature. It is very important to note that the $\alpha$ value is constant $(\sim 0.67)$ in all the temperature range, despite the emergence of conductance peak due to the $\mathrm{SC}$ regime at low $e V / k T$ regime below $\mathrm{T}=6 \mathrm{~K}$. This implies that the transition from the TLL behavior to the SC regime starts to occur only in the low $\mathrm{eV}$ regime, when the values of $e V$ are drastically lowered under constant low $k T$ values.

Here, in Fig. 6, we show that such a transition is actually possible on the light of the low-energy theories of doped MWNTs [10]. The two regimes are represented in the phase diagram of Fig. 6, which has been obtained following the approach of Ref. [10]. We observe that a minimum $N$ value per shell (10) in a MWNT is needed for the onset of the SC regime, and that subsequent peaks arise in the critical temperature as the Fermi level $\left(\varepsilon_{\mathrm{F}}\right) \operatorname{crosses}$ the top of a new subband (i.e., a Van Hove singularity). This result is in good agreement with the present experimental results, in which the $N_{B}$ value is below 2 at.\% with the small $N$ values leading to shift of $\varepsilon_{\mathrm{F}}$ in $\sim 0.3 \mathrm{eV}[20,21]$ and corresponding $\mathrm{T}_{\mathrm{c}}$ of $3 \sim 12 \mathrm{~K}$. 


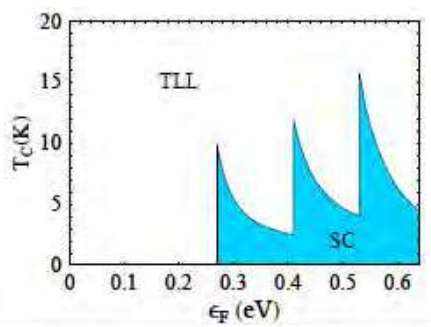

Fig. 6. Phase diagram of doped MWNTs (incorporating the average over different geometries of the shells) in terms of the doping level, represented by the shift in the Fermi energy $\varepsilon_{F}$ with respect to the charge neutrality point, and the temperature].

The characterization of doping in the MWNTs provides actually the clue to account for the SC regime. This appears as a consequence of the large screening of the Coulomb interaction, which arises in doped MWNTs, as in the case of CNT ropes [8], from the electrostatic coupling of a large number of $1 \mathrm{D}$ conduction channels. The effect is such that the long-range part of the Coulomb potential is largely suppressed, placing the system on the verge of a pairing instability. This takes place as the intertube Cooper-pair tunneling is progressively enhanced at low energies [10]. In this respect, the Cooper pairs do not find the obstruction that single electrons have to tunnel between the incommensurate CNT lattices of a MWNT. When intertube coherence can be established at some low temperature $\mathrm{T}_{\mathrm{c}}$ under a low eV value, the system undergoes the transition from the TLL to the SC regime as shown in Fig. 6. Although further quantitative investigation is expected, the present phenomena must shed light on understanding of interplay of SC with 1D electron correlations in $B$-CNTs.

\section{Superconductivity in thin films of boron-doped carbon nanotubes}

New carbon-based superconductors - calcium- intercalated graphite $\left(\mathrm{C}_{6} \mathrm{Ca}\right)$ and highly boron $(B)$ - doped diamond [23-25] - have been discovered recently and have attracted attention. It is well known that the small mass of carbon can promote high $\mathrm{T}_{\mathrm{c}}$ in Bardeen-Cooper-Schrieffer (BCS)-type SC and novel behaviors of SC can also be expected. As mentioned in section 1, the SC in a CNT is attracting significant attention [26-30] for the following reasons: (1) The curvature resulting from a small diameter can bring high $\mathrm{T}_{\mathrm{c}}$ due to a strong electron-phonon coupling [31]. (2) The alignment of the $\mathrm{E}_{\mathrm{F}}$ to a VHS can also lead to high $T_{c}$ due to the presence of an extremely large DOS [31]. (3) It can provide an insight into the 1D electron correlation [29, 32-36].

Here, it is also a well-known fact that effective carrier doping is crucial for realizing high- $\mathrm{T}_{\mathrm{c}}$ $\mathrm{SC}$ in any material. In fact, $\mathrm{SCs}$ in $\mathrm{C}_{6} \mathrm{Ca}$ and $B$-diamond have been associated with it. Carrier doping into CNTs has also been studied in many previous works [37-39]. Ref.[37] have reported a systematic study of SWNT bundles using the pulsed laser vaporization technique, in which the $B$ doping concentration $\left(N_{B}\right)$ is controlled by the amount of elemental $B$ mixed into a $\mathrm{Co} / \mathrm{Ni}$ catalyst impregnated targets. Evidence for substitutional $B$ doping and enhanced electronic DOS at the $\mathrm{E}_{\mathrm{F}}$ has been reported previously [37-39].

Nevertheless, experimental studies on the SC in carrier-doped CNTs are lacking. Although only I reported on SC in the $B$-MWNTs as explained in section 1, the boron doping was accidental and could not be in a controlled manner. This could be due to (1) difficulties 
associated with substitutional doping in CNTs (with diameter as small as $\sim 1 \mathrm{~nm}$ ) without compromising structural integrity of the hexagonal carbon network, (2) since the conventional size of Cooper pairs and the path of the Meissner shielding current cannot be smaller than $1 \mathrm{~nm}$, SC cannot be realized in an isolated single CNT [30], (3) in individual CNTs, strong 1D electron correlation phenomena tend to destroy Cooper pairs and the alignment of the $\mathrm{E}_{\mathrm{F}}$ to a VHS requires highly optimized doping rate. From these viewpoints, the assembled $B$-doped SWNTs with low $B$ concentration reported in this section are crucial for the observation of Meissner effcet [30,33,46]. Theoretical studies also suggests that SC can be realized in $B$-SWNTs due to small-dopant-induced $\left(\leq 0.4\right.$ at. \%) tuning of the $\mathrm{E}_{\mathrm{F}}$ with the VHS present in individual $(10,0)$ SWNTs [40] and also in carrier-doped MWNTs [36].

In this section, I report on SC in the thin films of $B$-SWNTs and clarify correlation with VHS in electronic DOS in 1D conductor. I report on the Meissner effect and show that only SWNT films consisting of low boron concentration leads to evident Meissner effect with $T_{c}=12 \mathrm{~K}$. I also show that a highly homogeneous ensemble of the SWNTs is crucial for realizing the Meissner effect. The first-principles electronic-structure study of the B-SWNT reveals strongly correlation of the boron concentration for Meissner effect with VHSs.

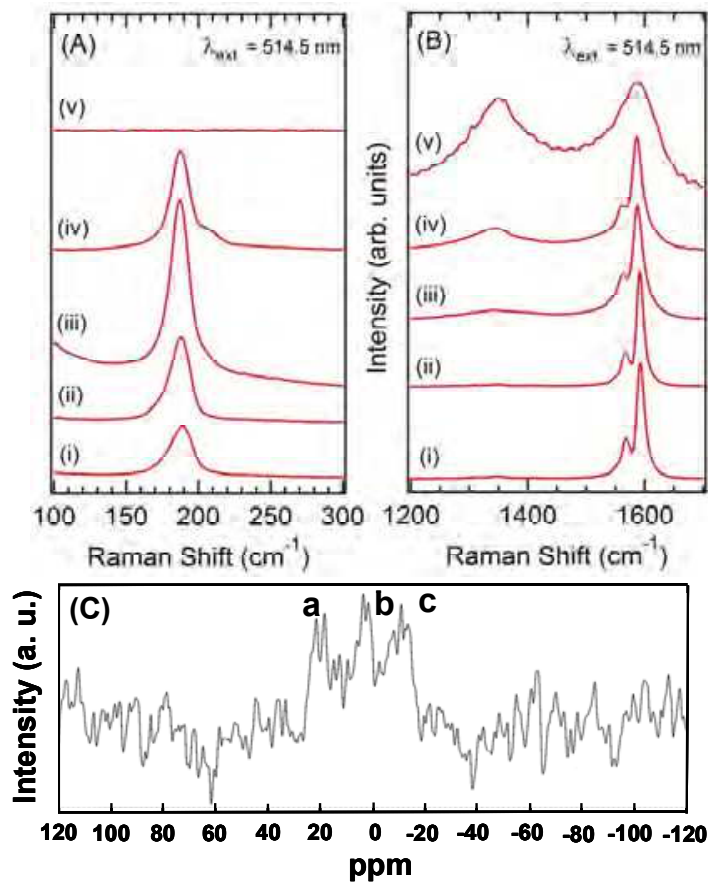

Fig. 7. (a)(b) Room temperature Raman spectra of semiconducting B-SWNT bundles synthesized from targets containing boron concentrations $\left(N_{B}\right)$ of $\sim$ (i) 0 (ii) 1.5 (iii)2.0 (iv) 3.0 (v) 4.5 atomic $\%$. An excitation wavelength $\lambda_{\text {ex }}=514.5 \mathrm{~nm}$ is used to probe the $B$-induced changes in the Raman spectra. The B-induced changes in (a) the RBM (present around 200 $\mathrm{cm}^{-1}$ ) and (b) the $D$ band (present around 1300-1350 $\mathrm{cm}^{-1}$ ) are shown. (c) Result of NMR measurement of $B$-SWNTs synthesized with $N_{B}=\sim 1.5$ at. \% target. Three peaks noted as a, $\mathrm{b}$, and c are evidently observed. 
Figure 7 (a) and (b) show the room-temperature Raman spectra of semiconducting SWNT bundles prepared from targets containing $B$ in the range $0<N_{B}<4.5$ at. \% (described above and in Ref. [37, 46]). The present catalytically $B$-doping method allows $B$ doping in low concentration in the SWNTs, avoiding destruction of carbon networks. The data set shown in Fig. 7 clearly reveals the following: (1) In Fig. 7(a), the intensity of the radial breathing mode $(R B M)$ varies nonlinearly with the $N_{B}$ and exhibits the highest values around $N_{B}=1.5$ and 2 at. \%. These intensity changes result from a change in the resonance conditions induced by the presence of $B$ in the SWNT lattice, which shifts the transition energies between the VHSs in the electronic DOS. (2) No SWNTs are present in the products prepared from targets with $N_{B}$ in excess of 4 at. \%, since the $R B M$ is absent in their spectra in Fig. 7(a). This decline is strongly correlated with the well-known saturation limit of $\sim 4$ at. \% for $N_{B}$ in graphite at $1200 \mathrm{~K}$. (3) In Fig. 7(b), a systematic increase in the intensity of the disorder-induced band ( $D$ band) with increasing $N_{B}$ is evident implying that a change in the degree of ordering in the hexagonal lattice of the SWNTs results due to the incorporation of $B$ in the lattice. Similar results are observed in the bundles of metallic SWNTs.

Figure 7(c) shows the result of nuclear magnetic resonance measurement (JNM-ECX400) of $B$-SWNTs synthesized target with $N_{B}=1.5$ at. \%. It evidently exhibits presence of three peaks $(\mathrm{a}, \mathrm{b}$, and $\mathrm{c})$. The peaks noted as $b$ and $c$ originate from chemical bonds between $B$ and carbon, in which the peak heights are highly sensitive to $B$ concentration in the target. In contrast, peak $a$ originates from $B$-oxide bond. The thermoelectric power (TEP) measurements also provide complementary evidence for the $B$-induced changes in the electronic properties since temperature dependent TEP studies revealed $p$-type characteristics [37]. These results collectively provide strong evidence for substitutional doping of $B$ in the SWNTs.
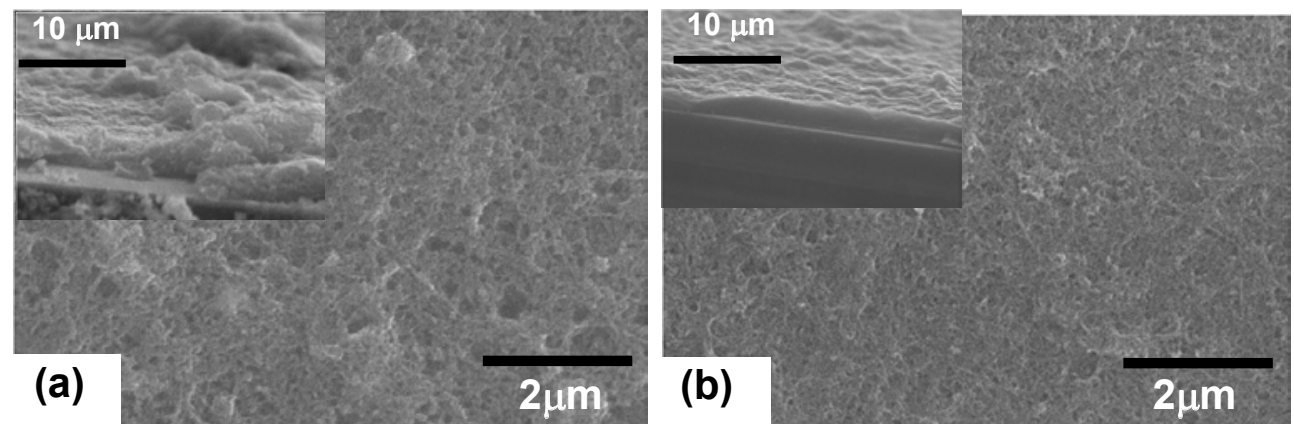

Fig. 8. Scanning electron microscope (SEM) images of thin films consisting of assembled $B$-SWNTs prepared on Si substrates. (a) Film fabricated without spin coating and (b) film fabricated by spin coating $(500 \mathrm{rpm})$. Insets: Cross-sectional SEM images of individual samples observed at a $30^{\circ}$ tilt.

The magnetization measurements of the thin films consisting of $B$-SWNTs [40] were performed using a superconducting quantum interference device (Quantum Design, MPMS) [24]. Figure 9 shows normalized magnetization $\left(M_{N}=M(T)-M(T=40 K)\right)$ as a function of the temperature $(T)\left(M_{N}-T\right.$ relationship) in field-cooled (FC) and zero-field -cooled (ZFC) regimes in the highly uniform film with $N_{B} \sim 1.5$ at. \% as shown in Fig. 8(b) . Evident drop in 
$M_{N}$ is observed below $\mathrm{T}_{\mathrm{c}}=12 \mathrm{~K}$ in the $\mathrm{ZFC}$ regime, and below $\mathrm{T}=\sim 8 \mathrm{~K}$ in the $\mathrm{FC}$ regime. Interestingly, this value of $T_{c}=12 \mathrm{~K}$ exactly agrees with the $T_{c}$ value for an abrupt resistance drop observed in the array of entirely end-bonded MWNTs [29]. The small $M_{N}$ drop observable at $T_{c}>12 \mathrm{~K}$ in the $\mathrm{ZFC}$ regime is due to diamagnetism of graphite structure of bundle of SWNTs and not associate with Meissner effect [41]. It is found that the magnitude of the drops observed in the $M_{N}-T$ relationship becomes considerable at magnetic field $(H)<$ $\sim 1400$ Oe as $H$ values increase. In contrast, it decreases for $H>\sim 1400$ Oe, and at $H=\sim 3500$ Oe, the magnitude becomes almost zero.

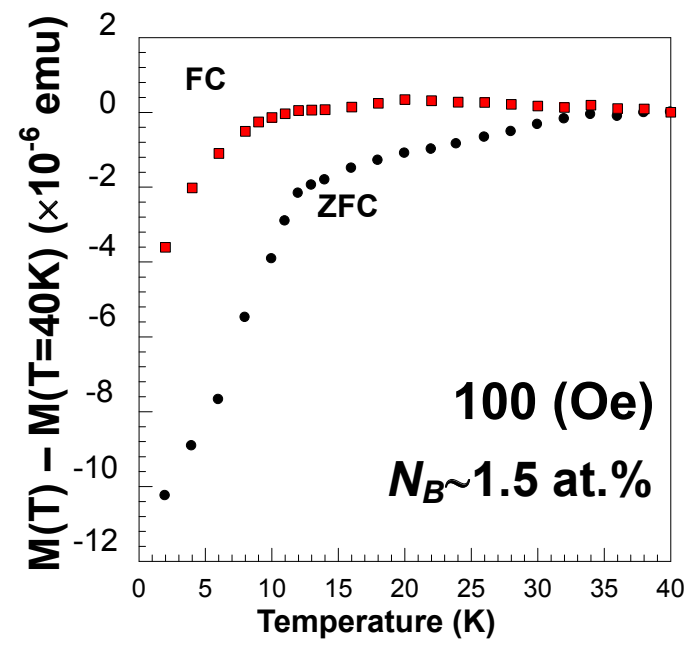

Fig. 9. Normalized magnetization as a function of temperature at magnetic fields $(H)$ of 100 Oe in FC and ZFC regimes in thin films of assembled B-SWNTs, which are synthesized from a target with $N_{B}=\sim 1.5$ at. \%. The films are prepared on a Si substrate by spin coating at $500 \mathrm{rpm}$.

Figure 10 shows the values of $M_{N}$ as a function of $H\left(M_{N}-H\right.$ relationship) for various $T$ in the ZFC regime in the sample shown in Fig. 9. At each temperature, $M_{N}$ values decrease at $H<$ $\sim 1400$ Oe, while they increase at $H>\sim 1400$ Oe. The magnitude of the drops decreases monotonically as $T$ increases. These behaviors observed in the shown $M_{N}-T$ and $M_{N}-H$ relationships are qualitatively in good agreement with the Meissner effect in type-II superconductors. 


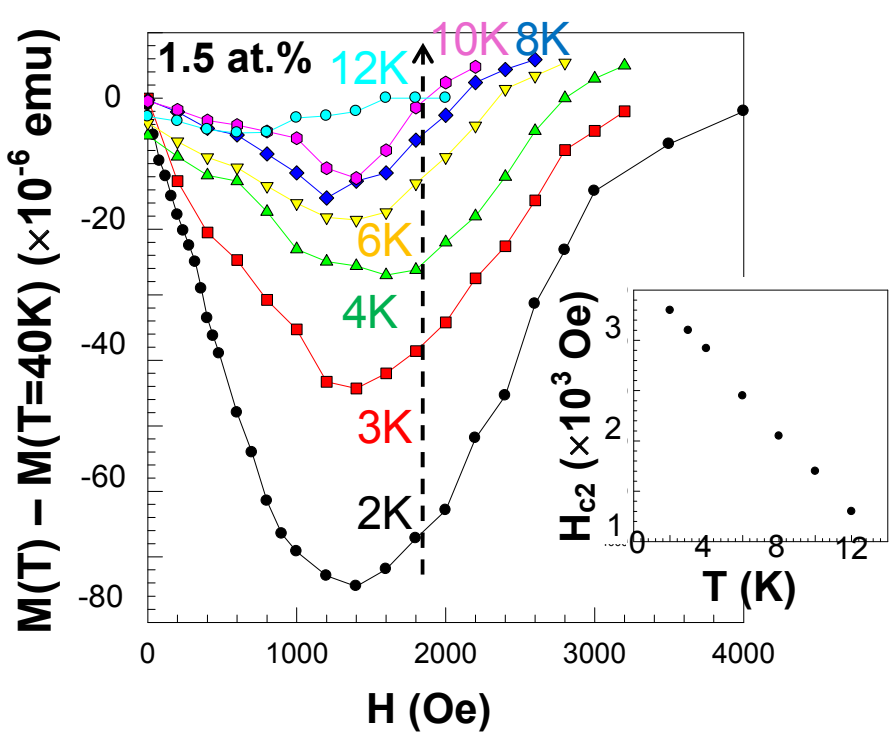

Fig. 10. Normalized magnetization vs. magnetic field for various temperatures in the sample shown in Fig. 9. Inset: Upper critical field $\left(\mathrm{H}_{\mathrm{c} 2}\right)$ vs. temperature relationship estimated from main panel. We determine $\mathrm{H}_{\mathrm{c} 2}$ at the $H$ value for which $M_{N}=0$ at each temperature by extrapolating the linear slope of the $M_{N}-H$ relationship for $H>\sim 1500$ Oe in main panel.

In order to confirm the Meissnser effect, we focus on the temperature dependence of the upper critical magnetic fields $\left(\mathrm{H}_{\mathrm{C} 2}\right)$ in the $M_{N}-H$ relationships shown in Fig. 10. The inset of Fig. 10 shows the result of the relationship between the estimated $\mathrm{H}_{\mathrm{c} 2}$ and $T$. The values of $\mathrm{H}_{\mathrm{c} 2}$ decrease linearly as the temperature increases. This result is also qualitatively in good agreement with the results for type-II superconductors. Residual $\mathrm{H}_{\mathrm{c} 2}$ around $\mathrm{T}_{\mathrm{c}}=12 \mathrm{~K}$ is attributed to diamagnetism of graphite as mentioned above.

From a quantitative viewpoint, the values of $\mathrm{H}_{\mathrm{c} 2}(\mathrm{~T}=0)=\sim 1700$ Oe can be estimated from the inset of Fig. 10 and the relationship

$$
\begin{gathered}
\mathrm{H}_{\mathrm{c} 2}(\mathrm{~T}=0)=-0.69\left(\mathrm{dH}_{\mathrm{c} 2} /\left.\mathrm{dT}\right|_{\mathrm{Tc}}\right) \mathrm{T}_{\mathrm{c}} \\
\mathrm{dH}_{\mathrm{c} 2} /\left.\mathrm{dT}\right|_{\mathrm{Tc}}=-200 \text { and } \mathrm{T}_{\mathrm{c}}=12 \mathrm{~K}
\end{gathered}
$$

The Ginzburg-Landau (G-L) superconductive coherence length

$$
\xi=\left[\Phi_{0} / 2 \pi \mathrm{H}_{\mathrm{c} 2}(\mathrm{~T}=0)\right]^{1 / 2}
$$

where $\Phi_{0}=\mathrm{h} / 2 \mathrm{e}$ is the quantum magnetic flux, can be estimated as $\xi=\sim 17 \mathrm{~nm}$ from the $\mathrm{H}_{\mathrm{c} 2}$ $(\mathrm{T}=0)=\sim 1700$ Oe. Further, the penetration length of the magnetic field 


$$
\lambda=\left(\mathrm{m}^{*} / \mu n_{\mathrm{s}} \mathrm{e}^{2}\right)^{1 / 2}
$$

is estimated to be of the order of $\sim 100 \mathrm{~nm}$. This $\lambda$ value is significantly larger than $\xi=\sim 17$ $\mathrm{nm}$. This result apparently supports the fact that the present films of $B$-SWNTs are type-II superconductors. Moreover, the value of $\xi=\sim 17 \mathrm{~nm}$ is in good agreement with $\xi=\sim 11 \mathrm{~nm}$ in the arrays of MWNTs [8], $\xi_{\mathrm{ab}}=13 \mathrm{~nm}$ in $\mathrm{C}_{6} \mathrm{Ca}[23,24]$, and $\xi=10 \mathrm{~nm}$ in $B$-doped diamond [25]. Therefore, we conclude that the $M_{N}-T$ drops shown in Fig. 3 and the corresponding $M_{N}-H$ relationship shown in Fig. 10 are attributed to the Meissner effect observed in type-II superconductors. The gradual and unsaturated $M_{N}$ drops in the Meissner effect imply inhomogeneous $B$ doping similar to the cases of $\mathrm{C}_{6} \mathrm{Ca}$ and $B$-diamond at early stage.

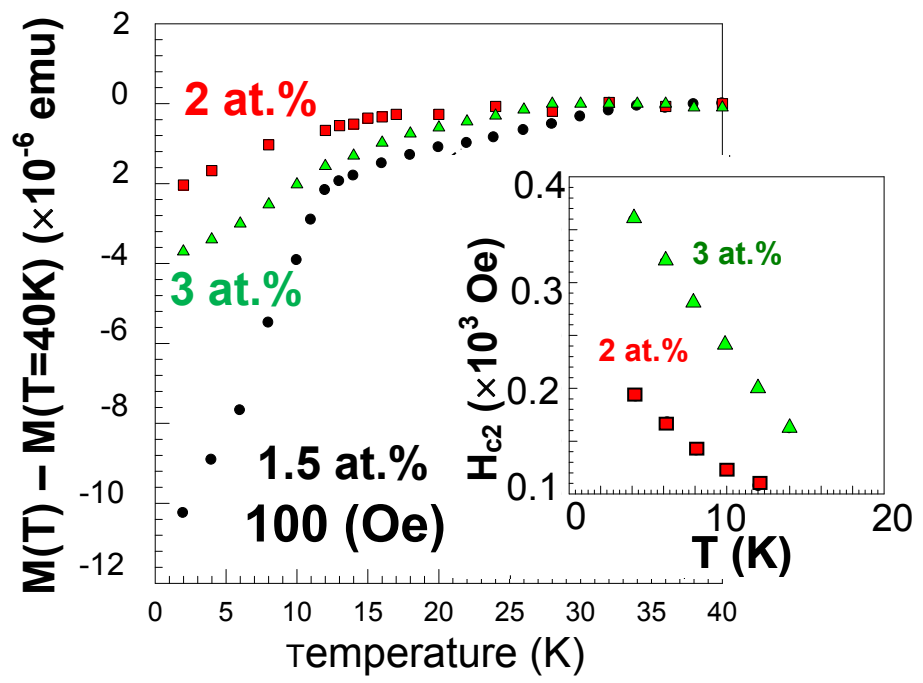

Fig. 11. Correlation of normalized magnetization drops with $N_{B}$ in targets. Contribution of graphite structure on magnetization is not subtracted.

Inset: $\mathrm{H}_{\mathrm{c} 2}$ vs. temperature relationships estimated from $M_{N}-H$ relationship in the thin films consisting of high $N_{B}$-value SWNTs (2 and 3 at. \%).

Here, we find that appearance of the Meissner effect is extremely sensitive to degree of uniformity of the thin $B$-SWNT films, which can be controlled by the conditions used in the spin coating process [40]. Figure 8 shows the SEM top view and cross-sectional images (observed at a tilt of 30 degree) of the thin films consisting of assembled $B$-SWNTs fabricated without spin coating (a) and by spin coating at $500 \mathrm{rpm}$ (b). The difference in the uniformities of the thin films shown in Figs. 8(a) and (b) is very evident. Further, in the case of spin coating at $1000 \mathrm{rpm}$, the extremely large number of rotations results in poor uniformity, similar to Fig 8(a). All 10 samples fabricated by the spin coating at $500 \mathrm{rpm}$ exhibit the evident Meissner effect, as shown in Figs. 9 and 10. In contrast, in the samples fabricated without spin coating and by spin coating at $1000 \mathrm{rpm}$, only one sample out of 20 exhibits the Meissner effect. This result suggests that the Meissner shielding current or a superconducting vortex is not confined to individual SWNTs but exists across assembled 
$B$-SWNTs in the thin films. Indeed, ref.[42] has predicted that Cooper pairs have a conducting probability across assembled SWNTs (with different chilarity) higher than that in individual electrons This suggestion is also consistent with $\xi=\sim 17 \mathrm{~nm}$ mentioned above, because $\xi$ corresponds to the diameter of Cooper pair and the diameter of individual SWNT is $\sim 1 \mathrm{~nm}$ at most.

Figure 11 shows the correlation of the $M_{N}-T$ and $M_{N}-H$ behaviors with the $N_{B}$ values in the catalyst in the ZFC regime. It clearly indicates strong sensitivity to $N_{B}$; i.e., (1) the $M_{N}$ drops become considerably smaller and ambiguous [41] in the samples with $N_{B}$ values of 2 at.\% and 3 at.\% as compared to those with $N_{B}=1.5$ at.\% and (2) the $M_{N}$ drop in the sample with $N_{B}=2$ at. \% is slightly more than that with $N_{B}=3$ at. \%. The $M_{N}-H$ relationships similar to that shown in Fig. 10 were found in the samples with $N_{B}=2$ and 3 at.\%. However, the $\mathrm{H}_{\mathrm{c} 2}$ values of the order of 100 Oe as shown in the inset of Fig. 11 are considerably smaller than those of the order of 1000 Oe shown in Fig. 10. The estimated values of $\mathrm{H}_{\mathrm{c} 2}(\mathrm{~T}=0)=\sim 280$ Oe and $\xi$ $=\sim 7 \mathrm{~nm}$ for the $N_{B}=3$ at.\% sample are also considerably smaller than the corresponding values in the $N_{B}=1.5$ at. $\%$ sample $\left(\mathrm{H}_{\mathrm{c} 2}(\mathrm{~T}=0)=\sim 1700\right.$ Oe and $\left.\xi=\sim 17 \mathrm{~nm}\right)$. These results imply that low $B$ concentration yields stronger SC behavior. This is mostly consistent with the intensity change in the $R B M$ as shown in Fig. 7, which exhibited the highest intensity around 1.5 and 2 at. \%.

This result is also qualitatively in good agreement with the first-principles electronic-structure study of the $B$-SWNT [43]. From the electronic band structure and DOS of $(10,0)$ SWNT with $N_{B}$ values of 0.83 at.\% to 2.50 at.\%, it can be predicted that the occurrence of SC should be sensitive to the correlation of the position of the Fermi level with that of the VHS, and that even lower $N_{B}$ value should be preferable for realizing SC as shown in inset of Fig. 12. Main panel of Fig. 12 shows the electronic structure of $\mathrm{BC}_{159}$ which corresponds to the $N_{B}$ value as low as 0.625 at.\% using the local-density approximation in the framework of the density functional theory [44]. It clarifies that the Fermi-level DOS of this system is considerably larger than those of higher $N_{B}$-value systems [45].

A resistance drop has not yet been detected owing to very high resistance $(>>\sim \mathrm{M} \Omega)$, because of (1) difficulty in establishment of good ohmic-contact between individual SWNTs and metal electrodes, (2) impossibility in having just a single $B$-SWNT aligned within the 1 $\mu \mathrm{m}$ electrode spacing since the maximum length of most SWNTs is $\sim 1 \mu \mathrm{m}$, and (3) difficulty in the entire end-bonding of the B-SWNTs [29] since the $B$-SWNTs are present inhomogeneously in the film.

The present results assure that further optimized $B$ doping into CNTs, and forming highly uniform ensemble of the $B$-SWNTs could lead to considerably high $\mathrm{T}_{\mathrm{c}}$ (e.g., up to $30 \sim 40 \mathrm{~K}$ as in alkali-metal-doped fullerenes and $\mathrm{MgB}_{2}$ ). Homogeneously assembled $B$-CNTs, which can provide weakly interacted CNTs (quasi-1D property) so as to maintain both the 1D properties (e.g., contribution of a VHS and strong curvature in one SWNT) and the 3D property (e.g., Meissner shielding-current path across assembled SWNTs), are promising as a novel structure which is expected to open doors to the fields of carbon-based SC. 


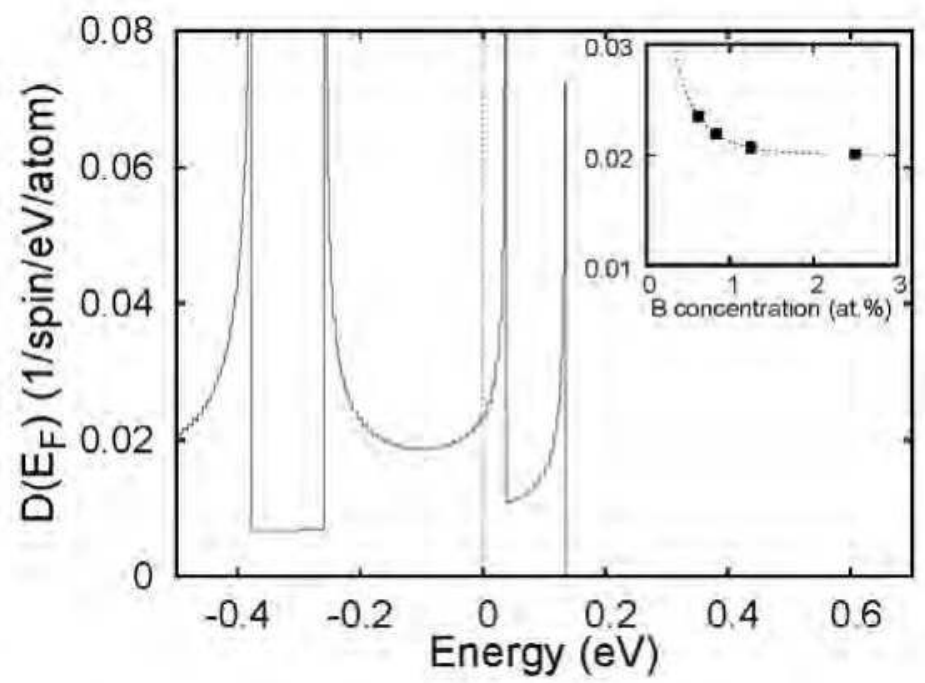

Fig. 12. Electronic density of states of individual $B$-doped semiconducting $S W N T, B_{159}$ obtained by using the local-density approximation in the framework of the density-functional theory. Plane-wave basis with the cutoff energy of 50 Ry is used. The host CNT is $(10,0)$ SWNT (i.e., the so-called zigzag nanotube) with the $N_{B}$ value as low as 0.625 at. \%. Energy is measured from the $\mathrm{E}_{\mathrm{F}}$.

Inset: Fermi-level density of states as a function of $N_{B}$.

\section{Pressure-induced superconductivity at $19 \mathrm{~K}$ in boron-doped Buckypapers}

As mentioned in section 2, the BCS-type superconductors are expected to exhibit a $T_{c}$, because of the high phonon frequency and Debye temperature of carbon atoms with low mass. Recently developed novel carbon-based superconductors (e.g., calcium-intercalated graphite and highly $B$-doped diamond [47-49]) have attracted significant attention from this viewpoint. However, the highest $T_{c}$ obtained is still now lower than $15 \mathrm{~K}$.

On the other hand, with CNTs, one expects much higher $\mathrm{T}_{\mathrm{c}}$, because (1) the curvature resulting from a small tube diameter (e.g., $<<1 \mathrm{~nm}$ ) can yield a strong coupling between the $\sigma-\pi$ electrons and RBM $[50,51]$ and (2) the alignment of the $E_{F}$ to a VHS in the electronic DOS (EDOS) can lead to an extremely high electronic density of states [51,52]. In the sections 1 and 2, I reported SC in the entirely MWNTs [53-55] and in the uniform thin films consisting of $B$--SWNTs [56]. The highest $T_{c}$ observed in these CNTs, however, is still now $12 \mathrm{~K}$.

In section 2 [56], I showed synthesis of B-SWNTs by mixing elemental $B$ powder into catalyst in order to intentionally generate charge carriers for SC and assembling them to thin films. We revealed that (1) B concentration $\left(N_{B}\right)$ as low as 1.5 atomic \% (at.\%) and (2) assembling into highly uniform thin films led to Meissner effect with the onset $T_{c}$ of $12 \mathrm{~K}$. I argued that this Meissner effect was realized by (1) the better alignment of $E_{F}$ to a VHS in the EDOS of the B-SWNT [52,56] and (2) providing of a loop current path for Meissner diamagnetism across multiple $B$-SWNTs by the abovementioned two factors, respectively 
$[54,56]$. In these viewpoints, assembling the B-SWNTs to film structure is crucial for realizing SC.

Recently, thin films consisting of SWNTs are attracting considerable attention for application to electrically-conductive transparent films and CNT -field effect transistors (FETs) [57]. Thus, thin films of B-SWNTs are also highly expected as superconducting transparent films. Here, in study of the above-mentioned previous $B-S W N T$ thin films, I had proposed the following measures for increasing $\mathrm{T}_{\mathrm{c}}$ : (1) employing lower $N_{B}$ values $\left(N_{B}<<1\right.$ at.\%); (2) using thinner B-SWNTs (e.g., diameter $<<1 \mathrm{~nm}$ ); (3) forming a dense assembly of thin films; and (4) applying pressure to the films.

In this section, I show fabrication of paper-like thin films consisting of pseudo two-dimensional network of weakly coupled B-SWNTs (the so-called Buckypaper) by sufficiently dissolving as-grown ropes of SWNTs and densely assembling them on silicon substrate. I show $\mathrm{T}_{\mathrm{c}}$ of $8 \mathrm{~K}$ under absent pressure in the Buckypaper and find enhancement of the onset $T_{c}$ up to $19 \mathrm{~K}$ by applying only a small pressure. An increase in the phonon frequency of the radial breathing phonon mode under applied pressure is also found and its correlation with the pressure-induced $\mathrm{T}_{\mathrm{c}}$ is discussed.

$B$-SWNTs were synthesized by the pulsed laser vaporization technique [56], in which $N_{B}$ was controlled by the amount of elemental $B$ mixed with the Co/Ni-catalyst-impregnated targets. Substitutional $B$ doping was confirmed by NMR (JNM-ECX400) and Raman spectra measurements. Conventional thin film samples of $B$-SWNTs and the present $B$-Buckypaper were prepared by solubilizing the $B$-SWNTs in dichloroethane solution with densities of $0.5-1 \mathrm{mg} / \mathrm{mL}$ and $3-5 \mathrm{mg} / \mathrm{mL}$ by centrifugation at $5000 \mathrm{rpm}$ for 1 day and $10000 \mathrm{rpm}$ for 2 days (Tomy; low-speed centrifuge) and ultrasonification (As One, US cleaner) for 2 days and 5 days, respectively. Then, the solutions were spin coated at $500 \mathrm{rpm}$ on a Si substrate.

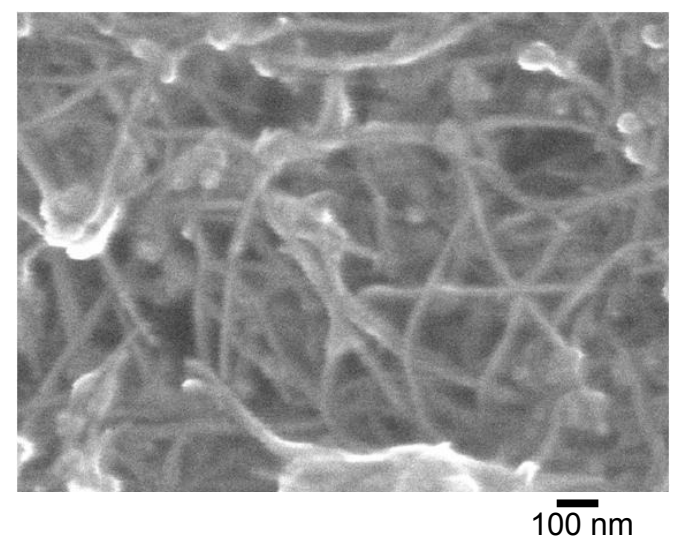

Fig. 13. (a) Top-view FESEM images of the conventional B-SWNT thin film fabricated by the conditions following our previous method. It consists of ropes of SWNTs.

Figure 13(a) shows the top-view field-emission scanning electron microscope (FESEM) images of the B-SWNTs thin films prepared by a previously reported method [56]. Although we tried to fabricate uniform thin films with dissolving ropes by the previous conditions, Fig. 13(a) reveals that the film still consists of thin ropes of as-grown $B$-SWNTs with $\sim 10 \mathrm{~nm}$ diameter. 
On the other hand, Fig. 13(b) shows the FESEM image of the present novel thin film consisting of $B$-SWNTs assembled densely after very strong centrifuges and ultrasonication of the SWNT solution. This figure clearly shows the absence of thick B-SWNT ropes (bundles) in the film and indicates that the film is sufficiently solubilized into ensemble of individual B-SWNTs. The fabrication conditions are harsher than those used for obtaining the film shown in Fig. 13(a). The film shown in Fig. 13(b) resembles a pseudo two-dimensional network of $B$-SWNTs deposited in the form of paper fibers. Such a SWNT network is known as a Buckypaper [57].

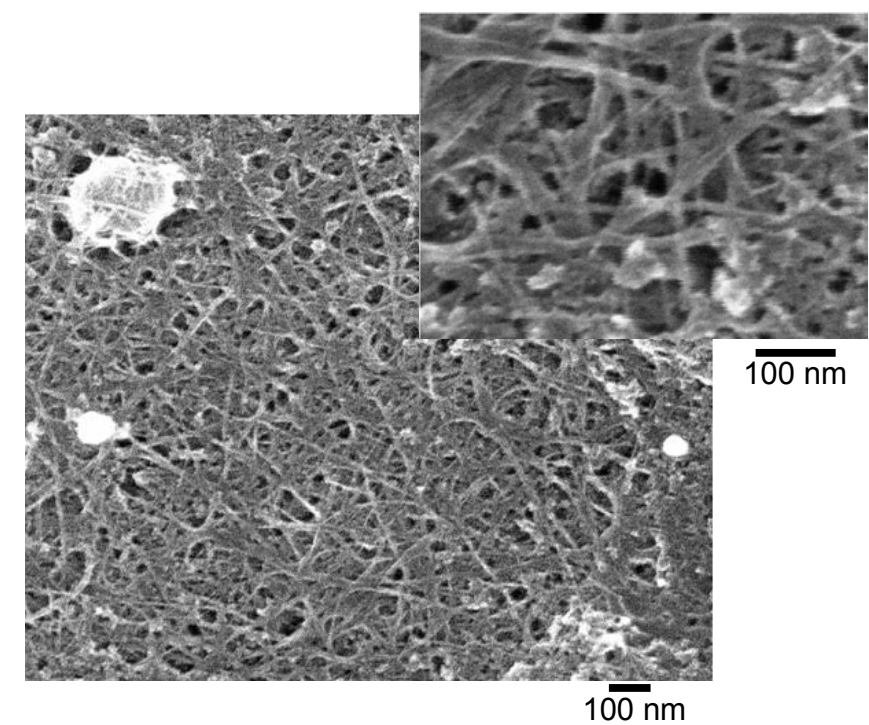

Fig. 13. (b) Top-view FESEM images of the present novel B-SWNT thin film (B-Buckypaper) fabricated using SWNTs sufficiently dissolved by strong ultrasonication. Ropes are unobservable.

We carried out magnetization measurements on the samples using a superconducting quantum interference device (Quantum Design, MPMS) and by embedding the samples in an oil-filled cell for applying pressure. Fig. 14 shows the normalized magnetization $\left(M_{N}=\right.$ $M(T)-M(T=30 \mathrm{~K}))$ of the sample shown in Fig. 13(a) as a function of temperature $(T)$ and pressure $(P)\left(N_{B} \sim 1.5\right.$ at.\%) at a magnetic field $(H)$ of 100 Oe in the zero-field-cooled (ZFC) regime. We could observe an apparent $\mathrm{T}_{\mathrm{c}}$ at $\sim 8 \mathrm{~K}$ and $P=0$. We have confirmed that diamagnetism observed below $\mathrm{T}_{\mathrm{c}}$ is attributed to Meissner diamagnetism for type-II SC on the basis of the magnetic field dependence of $M_{N}\left(M_{N}-H\right.$ relationship) at each temperature following our previous report [56], while the diamagnetism above $\mathrm{T}_{\mathrm{c}}$ that appears gradually from high temperatures is due to the graphitic structure of the $B$-SWNT ropes, as also explained in ref.[56]. Figure 14 indicates that the $\mathrm{T}_{\mathrm{c}}$ value does not change even at $P$ value as high as $3000 \mathrm{MPa}$. As $P$ increases further, graphitic diamagnetism becomes well pronounced. Then, $\mathrm{T}_{\mathrm{c}}$ corresponding to Meissner diamagnetism becomes no longer apparent. This tendency is also consistent with Fig. 13(a), which indicated presence of many ropes of $B$-SWNTs that produce strong graphitic diamagnetism. 
Meissner diamagnetism and graphite-originate diamagnetism were identified from measurements of $\mathrm{M}_{\mathrm{N}}-H$ characteristics following [56] and section 2. In the Meissner diamagnetism, magnitude of diamagnetism at each temperature increases with an increase in $H$, while it decrease above a critical $H$ value of $\sim 100$ Oe $\left(\right.$ lower $\mathrm{H}_{\mathrm{c}} ; \mathrm{H}_{\mathrm{cl}}$ ) and becomes zero at a $H$ value of $\sim 1000$ Oe (upper $\mathrm{H}_{\mathrm{c}} ; \mathrm{H}_{\mathrm{c} 2}$ ). We could confirm mostly linear relationship in the $\mathrm{H}_{\mathrm{c} 2}$ vs $T$ and estimate superconducting coherence length of $\sim 10 \mathrm{~nm}$ from the slope value, which is within a reasonable value compared with other carbon-based superconductors. Moreover, hysteresis in the $\mathrm{M}_{\mathrm{N}}-H$ curves could be observed on an increase and decrease in $H$. In contrast, in the graphite-originate diamagnetism, magnitude of the diamagnetism also increases with an increase in $H$. However, it does not decrease even at very high $H$ values, e.g., as large as $\sim 10000$ Oe. Moreover, this diamagnetism appeared from high temperatures (even from $\mathrm{T}=\sim 50 \mathrm{~K})$, very gradually. They have very different from those in Meissner effect. Figure 15 shows the $M_{N}-T$ relationships for the sample shown in Fig. 13(b) (B-doped Buckypaper ( $B$-Buckypaper) with $N_{B} \sim 1.5$ at.\%) at $P \leq 20 \mathrm{MPa}$ (Fig. 15(a)) and $P \geq 20 \mathrm{MPa}$ (Fig. 15(b)) at $H=100$ Oe in the ZFC regime. At $P=0, \mathrm{~T}_{\mathrm{c}}$ is evident at $\mathrm{T}=\sim 8 \mathrm{~K}$ in Figs. 15(a) and (c). Meissner diamagnetism at $T_{c} \leq 8 \mathrm{~K}$ and graphitic diamagnetism at $T_{c} \geq 8 \mathrm{~K}$ could be identified from the $M_{N}-H$ relationships, as in the case of Fig. 14.

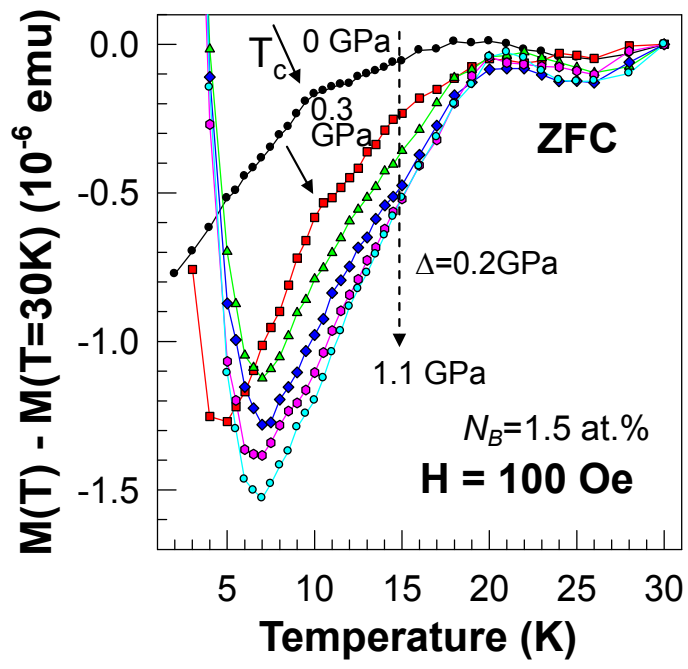

Fig. 14. Pressure dependence of normalized magnetization vs. temperature relationships for sample shown in Fig. 13(a) (conventional B-SWNT film) in ZFC regime.

In contradiction to Fig. 14, as $P$ increases, $\mathrm{T}_{\mathrm{c}}$ shifts to higher temperatures over two times (i.e., $\mathrm{T}_{\mathrm{c}}$ is $8 \mathrm{~K}$ at $0 \mathrm{~Pa}, 12 \mathrm{~K}$ at $10 \mathrm{MPa}$, and $19 \mathrm{~K}$ at $20 \mathrm{MPa}$ ). This behavior of $\mathrm{T}_{\mathrm{c}}$ is well apparent in Fig. 15(c), which was identical to Fig. 15(a) but has Y-axis in arbitrary unit. In contrast, as can be seen in Fig. $15(\mathrm{~b})$, as $P$ exceeds $20 \mathrm{MPa}, M_{N}$ increases almost over the entire temperature region, and the value of $\mathrm{T}_{\mathrm{c}}$ becomes unclear (i.e., possibly $\mathrm{T}_{\mathrm{c}}=10 \mathrm{~K}$ at 30 $\mathrm{MPa}$ and $8 \mathrm{~K}$ at $40 \mathrm{MPa}$ ). The overall $\mathrm{T}_{\mathrm{c}}-P$ relationship is shown in Fig. $3(\mathrm{~d})$. These behaviors are very different from those shown in Fig. 14 and suggest that the pressure dependence of the Meissner effect is highly sensitive to the structure of the $B$-SWNT assembly. 
Moreover, Fig. 15 shows that graphitic diamagnetism appearing above $\mathrm{T}_{\mathrm{c}}$ does not become stronger with an increase in $P$; in contradiction to that shown in Fig. 14. This behavior is consistent with the absence of the $B$-SWNT ropes in the $B$-Buckypaper as shown in Fig. 13(b) Furthermore, the $M_{N}-T$ behavior observed with increasing $P$ in Fig. 15(a)(b) is analogous to that observed in type-II SC with increasing $H$. This indicates a possibility of SC regions in the $B$-Buckypaper being compressed and becoming thin under applied pressure, because the aforementioned behavior can be observed when magnetic flux partially penetrates the superconductor as $H$ is increased.
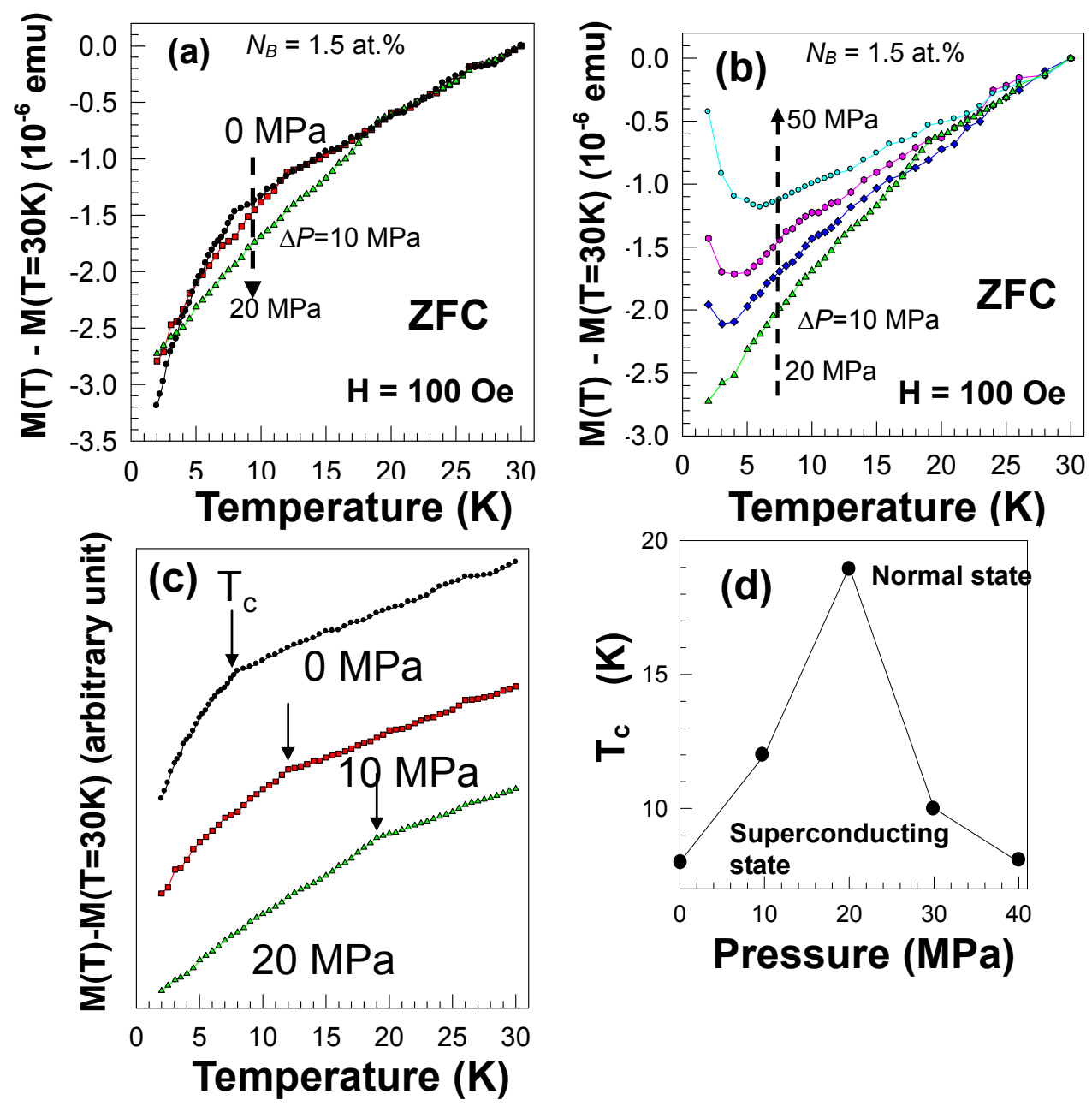

Fig. 15. (a), (b) Pressure dependence of normalized magnetization vs. temperature relationships of sample shown in Fig. 13 (b) (B-Buckypaper) at $\mathrm{P} \leq 20 \mathrm{MPa}$ (a) and $\mathrm{P} \geq 20 \mathrm{MPa}$ in zero-field cooled regime. (c) Identical to (a) but with Y-axis in arbitrary unit. (d) Pressure dependence of $\mathrm{T}_{\mathrm{c}}$. 
Here, we discuss the following two causes for the difference in the behavior of $T_{c}$ with an increase in $P$ (as shown in Figs. 14 and 15); (1) Increase in phonon frequency and (2) Change in charge transfer in the $B-C$ bonds. From the viewpoint (1), it is known that application of pressure to the SWNT ropes (bundles) compresses the triangular lattice of an SWNT rope and thus increase the phonon frequency. This in turn can cause an upshift in the frequency of the RBM and the $G$ band in the Raman spectra [58,59]. If a strong electron-phonon coupling exists when the frequency of the phonons increases under the application of pressure, $\mathrm{T}_{\mathrm{c}}$ should also increase in the manner shown in Fig. 15.

Figure 16 shows the pressure dependence of the Raman spectra for the sample shown in Fig. 13. Figures 16(a) and 16(b) correspond to the conventional film shown in Fig. 13(a), and Figs. 16 (c) and 16(d) correspond to the B-Buckypaper shown in Fig. 13(b). The pressure dependences of the peak positions observed in Figs. 16(a)-(d) are shown in Figs. 16(e) and 16(f). From Figs. 16(e) and 16(f), it is clear that at $P=0$, the peak positions observed for the $B$-Buckypaper are lower than those observed for the conventional film in both the RBM (Figs. 16(a) and 16(c)) and the G-band mode (Figs. 16(b) and 16(d)). This trend is consistent with the observation in Fig. 13, because the phonon frequency increases by intertubule van der Waals coupling of the SWNTs in a rope structure as shown in Fig. 13(a). This is also strong evidence for the sufficient dissolving of ropes in the $B$-Buckypaper, as shown in Fig. 13(b).

With an increase in $P$, all the peak positions show a monotonic upshift for ratios of $\sim 1$ $\mathrm{cm}^{-1} / 100 \mathrm{MPa}$ and $\sim 2 \mathrm{~cm}^{-1} / 100 \mathrm{MPa}$ in the RBM (Fig. 16(e)) and $\sim 0.7 \mathrm{~cm}^{-1} / 100 \mathrm{MPa}$ and $\sim 1$ $\mathrm{cm}^{-1} / 100 \mathrm{MPa}$ in the G-band mode (Fig. 16(f)) for the conventional film and the $B$-Buckypaper, respectively. These observations imply that the increase ratios for the $B$-Buckypaper are larger than those for conventional film in both modes. In particular, the RBM peak position for the $B$-Buckypaper shows the highest upshift ratio, which is twice the upshift ratio observed for the conventional film. Here, although the mean diameter of the present $B$-SWNTs is $\sim 1 \mathrm{~nm}$, the $B$-Buckypaper can include SWNTs with diameters smaller than $1 \mathrm{~nm}$ (e.g., $\sim 0.6 \mathrm{~nm}$ ). It is known that $\sigma-\pi$ electrons resulting from the $\mathrm{sp}^{3}$ hybrid orbitals in such thin SWNTs yield strong coupling with the RBM for formation of the loop current for Meissner diamagnetism [51]. Thus, the significant upshift in the RBM phonon frequency of $B$-Buckypaper shown in Fig. 16(e) can be origin for the pressure-induced $\mathrm{T}_{\mathrm{c}}$ in Fig. 15. Thus, the increase in $\mathrm{T}_{\mathrm{c}}$ seen in Fig. 15 can be mainly attributed to the increase in the RBM phonon frequency in the regions assembled by such thin $B$-SWNTs.

Also in the SWNT ropes shown in Fig. 13(a), pressure-induced phonon frequency could be observed in the RBM (Fig. 16(e)). However, the small increase ratio implies that the increase in phonon frequency is more significant in the $B$-Buckypaper. This is because the RBM is not restricted by van der Waals coupling in the individual SWNTs of the sufficiently solubilized $B$-Buckypaper and the phonon frequency can be easily induced under the application of pressure. 

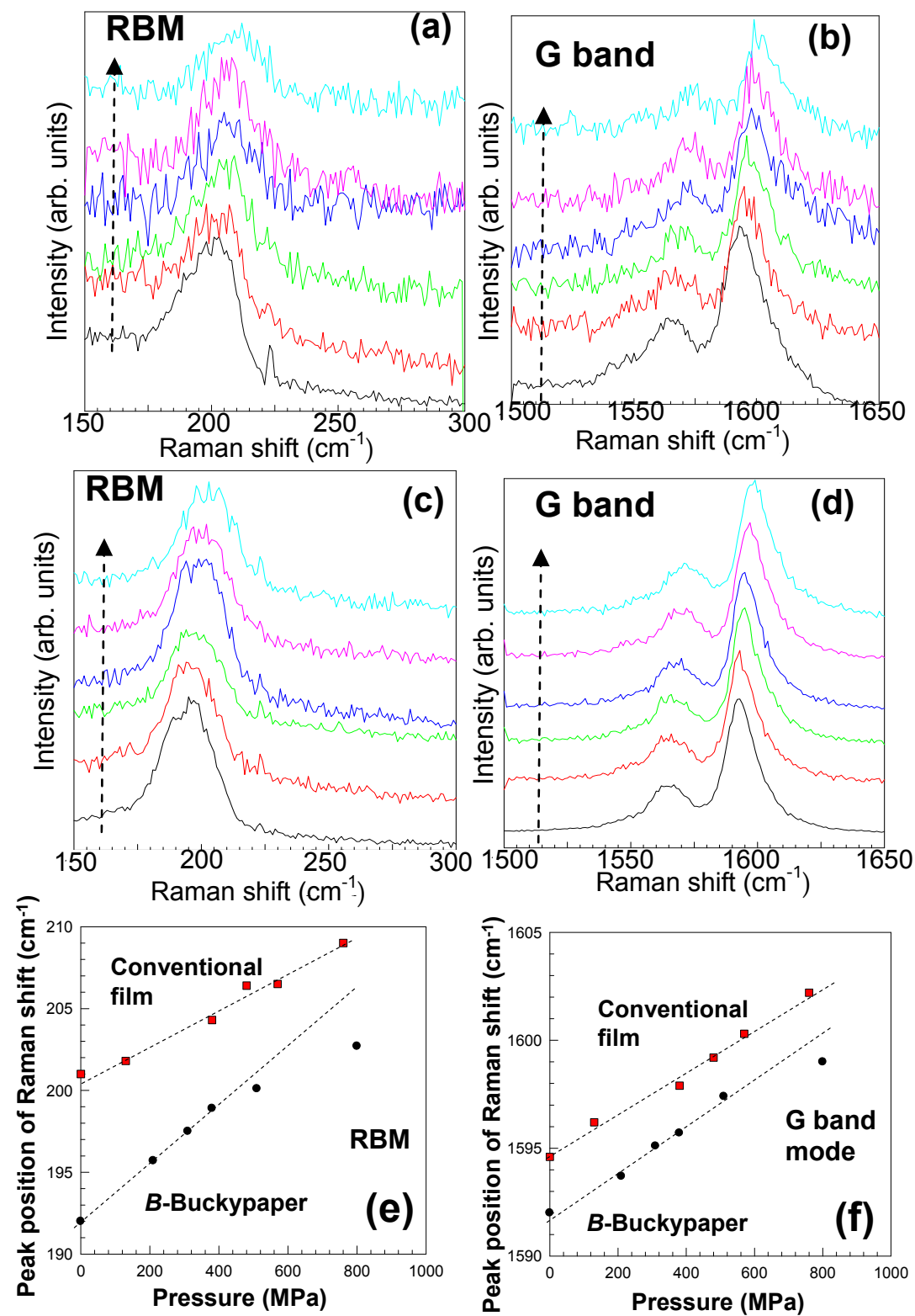

Fig. 16. Pressure dependence of Raman spectra for samples shown in Fig. 13; (a), (b) conventional film and (c), (d) B-Buckypaper. Spectra were measured by an irradiating Ar laser of $488 \mathrm{~nm}$ wavelength at room temperature. $P$ values are $0,130,380,480,570,760 \mathrm{MPa}$ for (a) and (b), and 0, 210, 310, 380, 510, $800 \mathrm{MPa}$ for (c) and (d) from the bottom to the top curves. 
Peak positions vs. pressure relationships in (e) RBM and (f) G-band mode. The peak positions are determined by data fitting based on Gaussian-Lorentzian curves in (a)-(d).

On the other hand, the following difficulties may arise for the abovementioned interpretation: (1) the applied pressure in Fig. 15(a) is as small as $20 \mathrm{MPa}$, which corresponds to an increase of $0.4 \mathrm{~cm}^{-1}$ in the phonon frequency; (2) in the case of the sample shown in Fig. 13(a), $T_{c}$ does not change in spite of the increase in the phonon frequency. However, these difficulties can be understood by difference in the electron -phonon coupling between the thin SWNTs and the ropes of SWNTs. With respect to difficulty (1), it has been predicted [51] that in very thin SWNTs, $T_{c}$ increases drastically even for a small increase in the phonon frequency owing to the strong electron -phonon coupling (the strong superconducting coupling strength $\lambda_{\mathrm{SC}}$ ). With respect to difficulty (2), it can be said that electron-phonon coupling is weak in the case of the sample shown in Fig. 13(a). This is due to reduction in the electron-phonon interactions in the rope structure, as it is known that intertube coupling in the rope structure weakens the electron-phonon coupling [51].

Here, from the standpoint (2) for the different $\mathrm{T}_{\mathrm{c}}$ behavior with an increase in $P$, changes in charge transfer caused by the $B-C$ bond modulation under the application of pressure can result in two effects for the increase in $\mathrm{T}_{\mathrm{c}}$.

One is the improved alignment of $\mathrm{E}_{\mathrm{F}}$ to a VHS by a decrease in the charge transfer. According to [56], with a decrease in $N_{B}\left(<1\right.$ at \%) and charge transfer, alignment of $\mathrm{E}_{\mathrm{F}}$ to a VHS is improved, resulting in a drastic increase in EDOS and in $\mathrm{T}_{c}$. When the $B-C$ bonds become weak and charge transfer decreases under the application of pressure, this phenomenon can appear in the present system. In fact, in $B$-doped diamond, $\mathrm{T}_{\mathrm{c}}$ decreases under high pressures [49], because of the decrease in charge transfer in the $B-C$ chemical bonds. Thus, a similar phenomenon can occur in the present $B$-Buckypaper, although its crystal structure is different from that of $B$-doped diamond. In our present experiment, a decrease in charge transfer and consequent shift of the $\mathrm{E}_{\mathrm{F}}$ position are small, because $P$ is small. However, an increase in the EDOS is extremely sensitive to the alignment of the $\mathrm{E}_{\mathrm{F}}$ to the VHS $[52,56]$ and can induce $\mathrm{T}_{\mathrm{c}}$ drastically.

Moreover, $B-C$ bonds can also be located inside a rope structure in the film shown in Fig. 13(a), where they are not significantly affected by the applied pressure. In contrast, the $B-C$ bonds in individual SWNTs are easily affected by the pressure, as in the case of the well-solubilized $B$-Buckypaper shown in Fig. 13(b). Consequently, the modulation in the $B$ - $C$ bonds can be the second cause for the increase in $T_{c}$ more or less. However, showing evidence for the modulation of $B-C$ bonds is indispensable.

The other is hole doping by an increase in the $B-C$ bonds and an increase in phonon frequency renormalized by hole-phonon interactions $[60,61]$. However, this is not the present case, because the charge transfer in the $B-C$ bonds is induced by applied pressure based on the above-mentioned discussion.

Further investigation of the causes for the pressure dependence of $T_{c}$ is necessary. However, the successful fabrication of the B-Buckypaper studied herein shows the feasibility of producing superconducting materials from light-mass atoms such carbon and boron. Moreover, the present results strongly imply a possibility for obtaining higher $T_{c}$ above $20 \mathrm{~K}$ in CNT ensembles by optimization of boron doping and film structures. 


\section{Possibility of quantum computation by utilizing carbon nanotubes}

\section{-Cooper pair splitting by Tomonaga-Luttinger liquid-}

Challenges for quantum computation (quantum bit) have been carried out in many material systems, such as superconductor- and semiconductor-quantum dots with single carrier charging effect, flux-controlled superconductor rings, Bose-Einstein condensed atoms and molecules, and NMR. No studies of quantum computation, however, have been reported in CNTs. It is well known that CNTs have strong electron spin coherence due to the unique electronic states and, hence, electron spins can be effectively transported through CNTs without losing the phase over a long distance and in a long time [62]. This characteristic gives a good chance for realizing quantum computation.

One of realistic ways for such challenges using CNTs is creation of quantum spin entangler (QSE) utilizing Cooper pairs injected from s-wave superconductor. Indeed, two theoretical studies have predicted possibilities of QSE by proposing a hybrid system of a superconductor and two CNTs within TLL states. They predicted separation of Cooper pairs into individual spins by strong repulsive Coulomb interaction of TLLs in CNTs [63][64]. As explained in section 1, TLL is non-Fermi liquid states consisting of a repulsive Coulomb interaction between electrons confined in 1D conductors and has been frequently reported in CNTs by observing power laws in relationships of conductance $(G)$ versus energies $(E)$ (i.e., $\mathrm{G} \propto \mathrm{E}^{\alpha}$ ) [65][66]. Strong repulsive electron-electron interaction in TLLs of CNTs can efficiently separate spin singlet of Cooper pairs, which are injected from s-wave superconductors, into individual spins and allows injection of the separated spins into different CNTs. Ref.[64] predicted differences in formulas for power laws between the cases of tunneling of separated Cooper pairs and non-separated Cooper pairs from a superconductor electrode to CNTs. Ref.[63] predicted that efficiency of the splitting of Cooper pairs strongly depends on tube spacing, dimension of superconductor, and strength of TLLs, etc.. Moreover, one experimental study reported on presence of possibly strong spin entanglement in spin singlet of Cooper pairs in CNTs by observing co-existence of Kondo singlet in Kondo effect and spin singlet in Cooper pairs in CNT quantum dots [67]. However, there are no any other experimental studies, which revealed behaviors of coherence and entanglement of Cooper pairs in CNTs, because it was difficult to efficiently inject Cooper pairs into CNTs owing to high interface resistances of CNTs/metal electrode junctions. In contrast, I successfully realized high-transparent interfaces of $\mathrm{Nb} / \mathrm{MWNTs}$ by utilizing end-bonded MWNTs synthesized in nanopores of alumina templates and found efficient injection of Cooper pairs (i.e., proximity-induced superconductivity) into CNTs [68]. In addition, I reported on possibilities of strong spin entanglement in Cooper pairs and separation of them into individual spins by TLLs [75].

Here, in this section, I report on (1) Evidence of spin separation in Cooper pairs by the TLLs and injection into different CNTs and (2) Possibility of molecular spin entangler by utilizing them. For the $1^{\text {st }}$ term, I report that injected Cooper pairs are separated by the TLLs following theories and individual spins exist in different CNTs. Magnetic-field dependence in these spins is for the first time investigated. For the 2nd term, I propose a hybrid system of one superconductive CNT and two Aharonov-Bohm (AB) ring CNTs within TLLs based on the $1^{\text {st }}$ and $2^{\text {nd }}$ terms. I explain that this system can be a good candidate for molecular spin quantum bit and entangler without decoherence problem. 

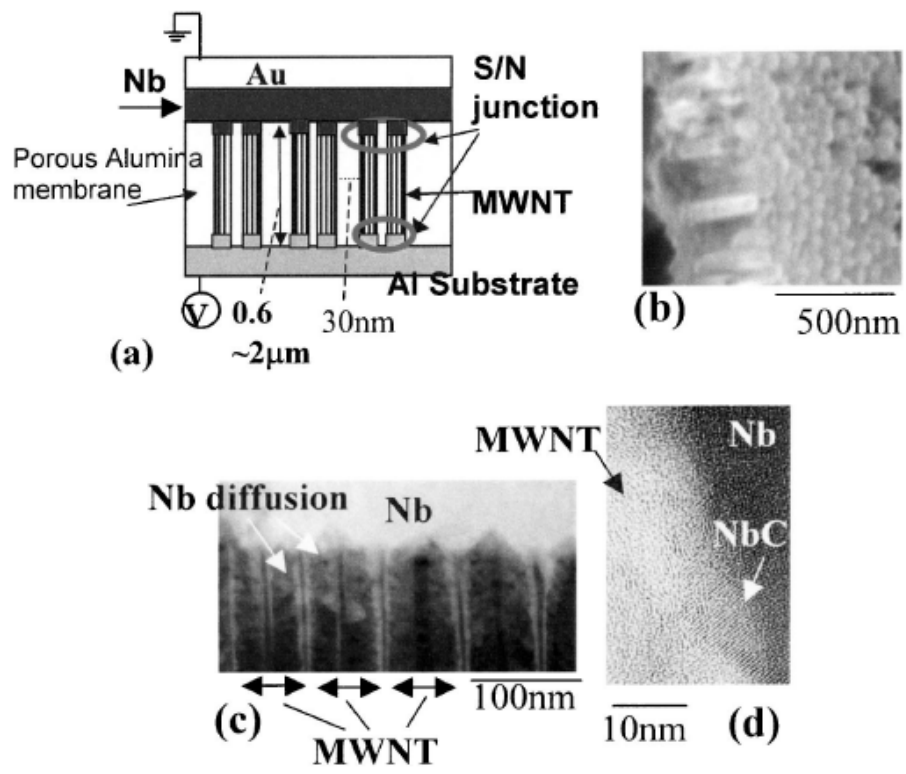

Fig. 17. (a) Schematic cross sections of an array of end-bonded $\mathrm{Au} / \mathrm{Nb} / \mathrm{MWNT} / \mathrm{Al}$ junctions, synthesized into the nanopores of alumina membranes by chemical vapor deposition of methanol gas, each with a thickness of $1 \mu \mathrm{m}$ for $\mathrm{Au} / \mathrm{Nb}$. $\mathrm{T}_{\mathrm{c}}$ of our $\mathrm{Nb}$ was about $8.1-9 \mathrm{~K}$ and $\mathrm{H}_{\mathrm{c}}$ was about $1500 \mathrm{G}$. The mean outer and inner diameters are 100 and $60 \mathrm{~nm}$, respectively, with a shell thickness as large as $20 \mathrm{~nm}$. (b) SEM overview image of a MWNT array, standing in the alu,ima membrane, implying high quality and open top portion of MWNTs. (c) High-angle annular dark-field image of a cross sectional TEM (CSTEM) around $\mathrm{Nb} / \mathrm{MWNT}$ interface array annealed by the optimal conditions, implying a high diffusion of $\mathrm{Nb}$ atoms. No diffusion of $\mathrm{Nb}$ into lower ends of the MWNT was also reconfirmed by EDX analyses. (d) High resolution CSTEM image in (b).

Figure 17(a) shows the schematic cross-sectional view of the sample structures (i.e., array of $\mathrm{Nb} /$ multi-walled CNs (MWNTs)/ Al junctions), which were prepared in array of nanopores of alumina templates. That's similar to the system in which I reported on intrinsic SC in section 1. However, in the present case, electrode metal is superconductor $(\mathrm{Nb})$ and the MWNT was synthesized by acetylene gas. I have reported on a variety of quantum phenomena in CNTs and metal nano-wires in this system [69]. This structure allows us to end-bond the MWNTs by evaporating metal electrode on the top ends of MWNTs (see Figs.17 (b) and (c)), as mentioned in section 1. This end-bonding has significantly enhanced interface transparency of metal electrode/CNTs junctions. I have successfully reported on proximity-induced superconductivity in this structure. 

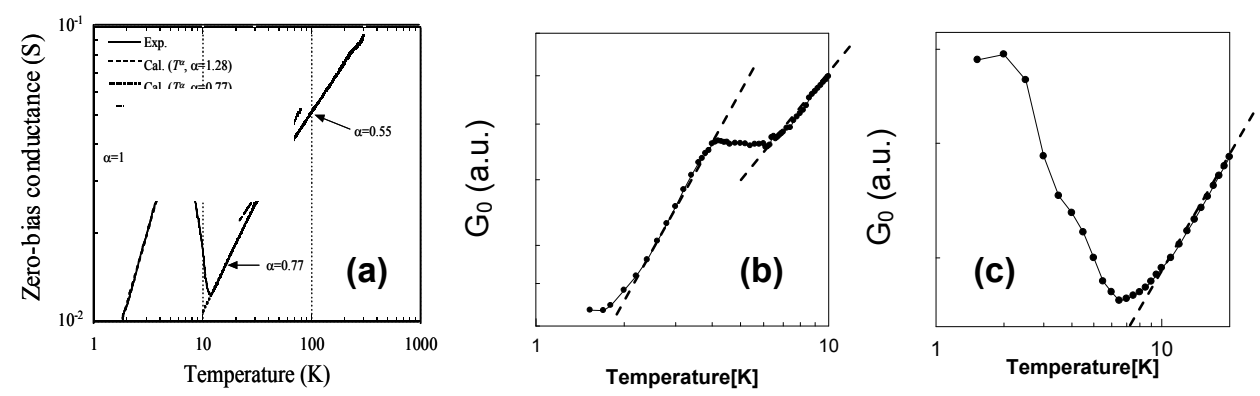

Fig. 18. Temperature dependence of the zero-bias conductance $G_{0}$ on a doubly logarithmic scale in $\mathrm{NbN} / \mathrm{MWNTs} / \mathrm{Al}$ junctions for different tube spacing with the $600 \mathrm{~nm}$-length MWNTs within a ballistic charge transport regime and TLL state. Tube spacing in arrays are (a) $20 \mathrm{~nm}$, (b) $30 \mathrm{~nm}$, and (c) $40 \mathrm{~nm}$. $\alpha$ in (a) is power value of power law behaviors in each temperature region.

Figure 18(a) shows the doubly logarithmic scales of zero-bias conductance versus temperature relationship in the sample with $\mathrm{L}_{\text {tube }}=600 \mathrm{~nm}$ and 20-nm tube spacing in a MWNT array. When the length of MWNTs for Fig. 18(a) was reduced below mean fee path $\sim 700 \mathrm{~nm}$ like this case, the MWNTs took a transition to a ballistic charge transport regime and showed power law behavior at temperatures $>\mathrm{T}_{\mathrm{c}} \sim 10 \mathrm{~K}$. Such power laws in the relationships of conductance vs. energies have been evidence for TLLs in both MWNTs [65] and SWNTs [66], with the following various powers $\alpha$.

$$
\alpha^{\text {bulk }}=\left(g^{-1}+g-2\right) / 8
$$

for tunneling from electrode to the bulk of CNTs and

$$
\alpha^{\text {end }}=\left(g^{-1}-1\right) / 4
$$

for tunneling from electrode to the ends of the CNTs

The observed correlation exponent $g$, the Luttinger parameter, is dictated by the strength of the electron-electron interaction, with ranges $g<1, g\rangle 1$ reflecting respectively its repulsive or attractive character. It determines $\alpha$, as low as $\sim 0.2$ stressed the presence of a strong repulsive Coulomb interaction in CNTs.

Because the observed power exponent $\alpha=\sim 0.8$ is in good agreement with $\alpha$ reported for Au-end junction [62], which just corresponds to our junction structure, this implies presence of TLLs. Correlation exponent $\mathrm{g}$ can be estimated to be $\mathrm{g}=\sim 0.25$ from the formula for (7) [65]. In contrast, this behavior disappears and conductance suddenly increases around $\mathrm{T}_{\mathrm{c}}$ and, then, it starts to decrease again at $\mathrm{T}<7 \mathrm{~K}$, following power law with the $\alpha \sim 1.28$ larger than $\alpha \sim 0.8$ mentioned above (Fig. 18(a)). This result stresses separation of Cooper pairs, injected from $\mathrm{Nb}$, into individual spins by TLLs of the MWNTs in accordance with the theory in [64].

Ref.[64] predicted that a tunneling probability $\Gamma_{\mathrm{AB}}$ of Cooper pairs from one superconductor to ends of two CNTs within TLLs is given by 


$$
\Gamma_{\mathrm{AB}}=\sim(\mathrm{eV} / \mathrm{h})(\mathrm{kT} / \varepsilon)^{[1 / \mathrm{g}-1] / 2}
$$

when Cooper pairs are separated into two spins, which tunnel into different two CNTs. We can obtain $\alpha=\sim 1.5$ by inserting $g=\sim 0.25$ into this formula. This $\alpha=\sim 1.5$ is approximately in agreement with the observed $\alpha=\sim 1.28$ in Fig. 18(a) and, hence, suggests possible splitting of Cooper pair by TLLs. In order to reconfirm this argument, the results, which were measured in the samples with tube spacing of $30 \mathrm{~nm}$ and $40 \mathrm{~nm}$, are shown in Fig. 18 (b) and (c), respectively. In Fig. 18(c), the conductance decrease, which appeared at T $<7 \mathrm{~K}$ in Fig. 18(a), entirely disappeared and only a conductance increase (i.e., proximity-induced superconductivity) is observable down to $\mathrm{T}=1.5 \mathrm{~K}$. In contrast, a conductance decrease following power laws appears at $\mathrm{T}<3 \mathrm{~K}$, which is lower than $\mathrm{T}=7 \mathrm{~K}$ in (a), in Fig. 18(b). These results are qualitatively in good agreement with the prediction by theory [63] as follows.

The theory [63] predicted inequality

$$
\mathrm{A}(2 \mu / \Delta)^{2 \gamma}<\left(1 / \mathrm{k}_{\mathrm{F}} \delta \mathrm{r}\right)^{\mathrm{d}-1}
$$

as the efficiency of Cooper pair splitting by TLLs in the system similar to ref.[64], where $d$ and $\Delta$ are the dimension of superconductor and superconducting gap, respectively, $\mu$ is the applied bias voltage between the superconductor and the leads, $\delta \mathrm{r}$ is the tube spacing, and $\gamma$ is the exponent for tunneling into the bulk of a single TLLs, which is proportional to $g$. As this inequality is easily satisfied, the efficiency of Cooper pair splitting increases. This inequality implies that increase in tube spacing $\delta \mathrm{r}$ results in a lower probability in Cooper pair splitting. Because emergence of only PIS in Fig. 18(c) for the largest $\delta$ r means no splitting of Cooper pairs, this agrees with the theory [63]. Shift of critical temperature for power law from $\mathrm{T}=7 \mathrm{~K}$ in Fig. 18 (a) to $\mathrm{T}=3 \mathrm{~K}$ in Fig. 18 (b) also supports this argument. Consequently, the results in Fig. 3 are qualitatively in good agreement with this theory [63].

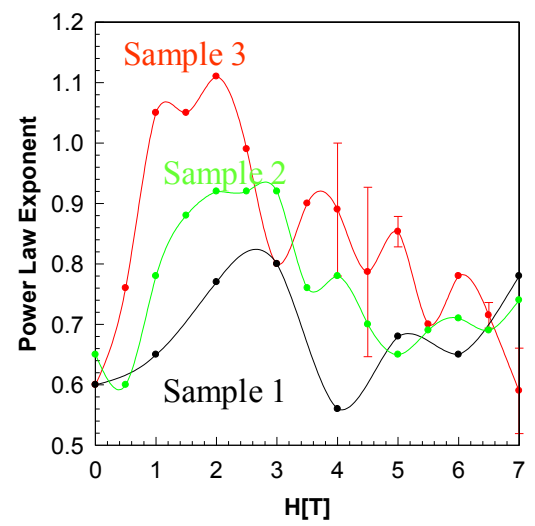

Fig. 19. The value of power $\alpha$ in power laws at low temperatures in Fig. 18(a) as a function of magnetic field for different three samples.

Figure 19 shows the values of powers $\alpha$ in the low temperature region in Fig. 18(a) as a function of magnetic fields $(\mathrm{H})$. Power laws still survive, even if $\mathrm{H}=7 \mathrm{~T}$ is applied. The value 
of $\alpha$ is sensitive to $H$. We find that the value of $\alpha$ increase as $H$ starts to increase at $H<\sim 3 T$, while it monotonically decreases at $\mathrm{H}>\sim 3 \mathrm{~T}$ with exhibiting small oscillative behaviors.

Ref.[71] predicted that the value of $\alpha$ monotonically decreases as $H$ increases in TLLs within a short range Coulomb interaction, because spins with opposite momenta localize at opposite ends of a CNT due to the applied $\mathrm{H}$ and, hence, a repulsive Coulomb interaction between the electrons is reduced and the value of $\mathrm{g}$ increases, resulting in a decrease in $\alpha$. This theory is qualitatively in good agreement with the decrease in $\alpha$ in the region of $\mathrm{H}>3 \mathrm{~T}$ in Fig. 19. Hence, this can be evidence for TLLs. Because the tube length of about $600 \mathrm{~nm}$ is large enough for a short-range interaction and Coulomb interaction is easily reduced by the edge localization, this agreement is appropriate.

In contrast, the increase in $\alpha$ at $\mathrm{H}<3 \mathrm{~T}$ cannot be explained by this theory. The difference between conventional TLLs described by theory [71] and our system is at least that separated Cooper pairs are injected into the ends of MWNTs in our case. Hence, this increase in $\alpha$ at low fields can be attributed to this difference. We propose one interpretation for this result as follows. When separated Cooper pairs are injected as individual spins into different MWNTs at $\mathrm{H}=0 \mathrm{~T}$, momentum of spins are highly aligned in comparison to those in single spins injected from normal conductor electrodes. When two spins separated from Cooper pair are still entangled state, this alignment is much stronger. Moreover, MWNTs have spin coherence. Hence, when low fields are applied to such highly aligned spins, electron-electron interaction may be enhanced, resulting in the increase in $\alpha$ as shown in

Fig. 19. As the fields increase further and electron start to localize, this situation is destroyed and the value of $\alpha$ decreases. Therefore, this can be evidence for entangled spins existing in different MWNTs.

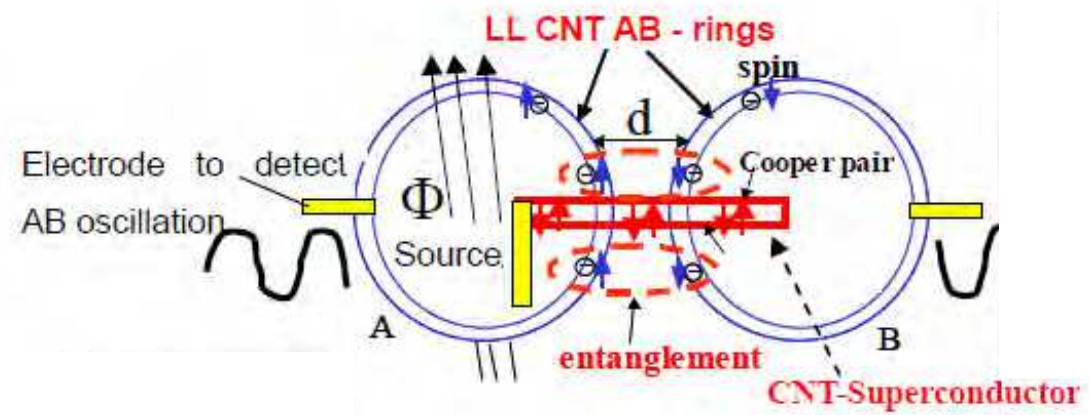

Fig. 20. An example of molecular quantum spin entangler; A hybrid system of one superconductive CNTs and two Aharonov-Bohm (AB) ring CNTs within TLLs. Cooper pairs, which are injected from the SC-CNT to AB CNT-ring, are separated to individual spins by repulsive Coulomb interaction of TLL state and injected to different rings. Strong spin coherence and entanglement of Cooper pairs in CNTs might realize spin quantum bit with dissolving decoherence problem.

Here, Fig. 20 shows one of examples of molecular QSE, based on a hybrid system of one superconductive CNT and two normal conductive CNTs within TLL states discussed above. Normal conductive CNTs form $\mathrm{AB}$ rings in this system. Cooper pairs are efficiently separated to individual spins by TLLs in the AB-ring CNTs, because superconductor CNT is within $d=1$ regime and following the inequality (9) [63], efficiency of Cooper pair splitting 
can be enhanced by reduction of dimension of superconductor (d). The separated spins are injected into the different $\mathrm{CNT}-\mathrm{AB}$ rings with opposite spin momenta maintaining memory of spin singlet. As shown in Fig. 19, electron-electron interaction is enhanced at low fields. Hence, when a low magnetic field is applied so as to penetrate only into one $A B$ ring, $A B$ oscillation can be very sensitive to the applied fields. If the two spins existing in the different $A B$ rings are still within entangled state, spin existing in the other $A B$ ring should show opposite-phase $A B$ oscillation even without applying magnetic fields. Therefore, one can investigate spin entanglement by simultaneously observing phase of these two $A B$ oscillations. If Cooper pairs in CNTs actually have a strong entanglement [67][75], separated spins retain a strongly entangled state crossing the different CNTs. This results in realization of good molecular QSE. One can explore this by increasing AB ring spacing.

Therefore, CNTs can be good candidates for realizing molecular quantum computation with strong spin coherence and entanglement.

\section{Acknowledgements}

The article described here was the results from many collaborators. J.H. sincerely acknowledges H.Shinohara, H. Fukuyama, J. Akimitsu, T. Ando, S.Maruyama, S. Saito, R.Saito, S.Tarucha, Y.Iye, N. Nagaosa, H.Takayanagi, M.Tsukada, Y.Hasegawa, T.Sugai, S. Bandow, M.Dresselhaus, A. M. Rao, J. Gonzalez, H. Bouchiat, C. Schoenenberger, D. Loss, R. Egger, A. Bachtold, P.E.Lindelof, J.-P. Leburton, J.Reppert, P.Recher, T.Koretsine, T.Nishio, H.Sano, Y.Awano, H.Nihey S.Okazaki, Y. Muranaka, O. Kamo, T.Nakanishi, T.Akazaki, W.Izumida, H.Sugiura, M.Tachibana, and my students and stuff.

\section{References}

1. M.Kociak, et al., Phys.Rev.Lett. 86, 2416 (2001)

2. I.Takesue, J.Haruyama et al., Phys.Rev.Lett.96, 057001 (2006). Power laws have been also discussed from viewpoints of Coulomb blockade coupled with its external electromagnetic environment (i.e., MWNT acts as a LC transmission line connected to tunnel junctions) [4] and also one-dimensional localization. However, the present case does not correspond to these models, because the calculation result of our MWNT as LC transmission line does not agree with the observed power laws and also the present interplay of power laws with SC cannot be understood by these models.

3. Z.K.Tang, et al., Science 292, 2462 (2001)

4. A.Bachtold, et al., Phys.Rev.Lett. 87, 166801 (2001)

5. M.Bockrath, et al., Nature 397, 598 (1999); Z.Yao, et al., Nature 402, 273 (1999)

6. R.Egger, Phys.Rev.Lett. 83, 5547 (1999)

7. I.Takesue, J.Haruyama, H.Takayanagi et al., Physica E 24, 32 (2004); P.Recher et al, Phys.Rev.B 65, 165327 (2002); C.Bena et al., Phys.Rev.Lett.89, 037901 (2002)

8. J.Gonzalez, Phys.Rev.Lett. 88, 076403 (2002); A.Sedeki, et al., Phys.Rev.B 65, 140515(R) (2002); J.Gonzalez, Phys.Rev.Lett. 87, 136401 (2001)

9. D.Loss and T.Martin, Phys.Rev.B 50, 12160 (1994-II)

10. E.Perfetto and J.Gonzalez, Phys.Rev.B 74, 201403(R) (2006) 
11. R.Egger and A.O.Gogolin, Phys.Rev.Lett. 79, 5082 (1997)

12. C.Kane, L.Balents et al., Phys.Rev.Lett. 79, 5086 (1997)

13. M.Monteverde, G.Garbarino et al., Phys.Rev.Lett. 97, 176401 (2006)

14. A.De Martino and R.Egger, Phys.Rev.B 67, 235418 (2003), Phys.Rev.B 70, 014508 (2004)

15. K.McGuire, et al., Carbon 43, 219 (2005)

16. K.Liu, et al., Phys.Rev. B 63, 161404R (2001)

17. Experimental observation of TLL states and its confirmation by theory requires $v(E)$ in CNTs [1], while SC transition may not favor a tunnel junction. However, Ref [12] predicted that even attractive Coulomb interactions could lead to power law behaviors along with perfect transmission of Cooper pairs via tunnel junction. Thus, the present result is consistent with this theory.

18. $v_{F}\left(=h k_{F} / m^{*}\right)$ and $L$ are the Fermi velocity and the tube length of the $1 \mathrm{D}$ conductor, respectively. The previous studies on TLL states in CNTs reported that the saturation regimes fall onto a universal curve in any temperature ranges.

19. N. Murata, J.Haruyama, S.Maruyama, H.Shinohara et al., Phys. Rev. B 71, 081744 (2007)

20. N. Murata, J.Haruyama, J.Reppert, A.Rao et al., Phys.Rev.Lett. 101, 027002 (2008).

21. T. Koretsune, S. Saito, Phys. Rev. B 77, 165417 (2008)

22. As the critical temperature of $6 \mathrm{~K}$ for the appearance of the deviation is in excellent agreement with that from Fig. 4(b), the SC regime potentially exists even at $3 \mathrm{~K}<\mathrm{T} \leq$ $6 \mathrm{~K}$, though the conductance peak is not seen there due to the obstruction by the TLL regime.

23. T. E. Weller et al., Nature Physics 1, 39 (2005).

24. N. Emery et al., Phys. Rev. Lett. 95, 87003 (2005).

25. E. A. Ekimov et al., Nature 428, 542 (2004).

26. M. Kociak et al., Phys. Rev. Lett. 86, 2416 (2001).

27. Z. K. Tang et al., Science 292, 2462 (2001).

28. M. Ferrier et al., Phys. Rev. B 73, 094520 (2006).

29. I. Takesue, J.Haruyama H.Shinohara et al., Phys. Rev. Lett. 96, 057001 (2006). "The $\mathrm{T}_{\mathrm{c}}=$ $12 \mathrm{~K}^{\prime \prime}$ can be a magic number. The common $\mathrm{T}_{\mathrm{C}}=12 \mathrm{~K}$ implies presence of common origins in ensemble of $B$-CNTs (e.g., the best alignment of $\mathrm{E}_{\mathrm{F}}$ to a VHS under this $N_{B}$ value). In contrast, because $\mathrm{C}_{6} \mathrm{Ca}$ has a different structure, the agreement of $T_{c}$ may be occasional.

30. N. Murata, J.Haruyama, S.Maruyama, H.Shinohara et al., Phys. Rev. B 71, 081744 (2007).

31. R. Barnett et al., Phys. Rev. B 71, 035429 (2005).

32. M. Matsudaira, J.Haruyama., H.Shinohara et al., Physica E 40/7, 2299-2304 (2008)

33. M. Matsudaira, J.Haruyama., et al., Phys. Rev. Lett. (2008) in submission.

34. I.Takesue, et al., Physica E 24, 32 (2004); P. Recher, D. Loss, Phys. Rev. B 65, 165327 (2002);

C. Bena et al., Phys. Rev. Lett. 89, 037901 (2002).

35. J. Gonzalez, Phys. Rev. Lett. 88, 076403 (2002).

36. E. Perfetto, J. Gonzalez, Phys. Rev. B 74, 201403(R) (2006).

37. K. McGuire et al., Carbon 43, 219 (2005).

38. K. Liu et al., Phys. Rev. B 63, 161404R (2001).

39. S. Bandow, S. Numao, S. Iijima, J. Phys. Chem. C 111, 11763 (2007).

40. http://www.ee.aoyama.ac.jp/Labs/j-haru-www/ 
41. Graphite diamagnetism in ensemble of SWNTs very gradually appears in $M-T$ curves from high temperatures (e.g., $>50 \mathrm{~K}$ ) even in undoped samples and the amplitude does not decrease in $M$ - $H$ features even at high magnetic fields (e.g., > 2000 Oe). They are very different from those in Meissner effect observed here. However, because the component is very small in the samples shown here and different by respective samples, we could not entirely delete it in this study.

42. J.Gonzalez, Phys.Rev.Lett. 88, 076403 (2002)

43. T. Koretsune, S. Saito, Phys. Rev. B 77, 165417 (2008)

44. http://www.pwscf.org/

45. Because we have measured SWNTs with $N_{B} \geq 1.5$ at. \% . in the present experiment, $N_{B}=1.5$ at.\% is the lowest value and, hence, it is qualitatively consistent with the theory. For quantitative agreement, the present theory should take into account influence of "ensemble" of B-SWNTs. Here, if the SWNTs are undoped, the difference in chirality (i.e., semiconducting / metallic behaviors) of the respective SWNTs is important for producing SC. In contrast, in carrier-doped SWNTs, SC is no longer sensitive to them, because one can freely control the position of $\mathrm{E}_{\mathrm{F}}$.

46. N.Murata, J.Haruyama, M.Matsudaira, J. Reppert, A.Rao, T.Koretsune, S.Saito, Y.Yagi, Phys.Rev.Lett. 101, 027002 (2008)

47. T. E. Weller et al., Nature Physics 1, 39 (2005).

48. N. Emery et al., Phys. Rev. Lett. 95, 87003 (2005).

49. E. A. Ekimov et al., Nature 428, 542 (2004).

50. M. Kociak et al., Phys. Rev. Lett. 86, 2416 (2001).

51. R. Barnett, E. Demler et al., Phys. Rev. B 71, 035429 (2005).

52. T. Koretsune, S. Saito, Phys. Rev. B 77, 165417 (2008)

53. I. Takesue, J. Haruyama H. Shinohara, S. Maruyama et al., Phys. Rev. Lett. 96, 057001 (2006).

54. N. Murata, J. Haruyama, S. Maruyama, H. Shinohara et al., Phys. Rev. B 71, 081744 (2007).

55. M. Matsudaira, J. Haruyama, S. Maruyama, H. Shinohara et al., Physica E 40/7, 2299 (2008).

56. N. Murata, J. Haruyama, J. Reppert, A.M. Rao, T. Koretsune, S. Saito, et al., Phys. Rev. Lett. 101, 027002 (2008).

57. J.A. Fagan, M.L. Becker, J. Chun, E.K. Hobbie, Advanced Materials 20, 1609 (2008).

58. U.D. Venkateswaran, A.M. Rao, R.E. Smalley, P.C. Eklund at al., Phys. Rev. B 59, 10928 (1999-II)

59. U.D. Venkateswaran, A.M. Rao, P.C. Eklund et al., Phys. Stat. Sol. (b) 223, 225 (2001)

60. J.C. Tsang, M. Freitag, V. Perebeinos, J. Liu, Ph. Avouris, Nature Nanotechnologies 11, 321 (2007).

61. A.M. Rao, P.C. Eklund, S. Bandow, A. Thess, R.E. Smalley, Nature 388, 257 (1997).

62. L.Langer, V.Bayot, J.-P.Issi, et al., Phys.Rev.Lett 76, 479 (1996)

63. P.Recher and D. Loss, Phys. Rev. B 65, 165327 (2002);

64. C.Bena et al., Phys.Rev.Lett.89, 037901 (2002)

65. A.Bachtold, C.Shonenberger, et al., Phys.Rev.Lett. 87, 166801 (2001); M.R.Buitelaar, et al., Phys.Rev. Lett. 88, 156801 (2002); A.Bachtold, et al., Nature 397, 673 (1999);

66. M.Bockrath, et al., Nature 397, 598 (1999); H.Ishii, H.Kataura, et al., Nature 426, 540 (2003)

67. M.R.Buitelaar, T.Nussbaurner, C.Shonenberger, Phys.Rev. Lett. 89, 256801 (2002) 
68. J.Haruyama, et al., Phys.Rev.B 68, 165420 (2003); Appl.Phys.Lett. 84, 4714 (2004); Microelectronics Journal 34, 537 (2003); Physica C 408, 85 (2004)

69. J. Haruyama, et al., Phys. Rev. B 65, 33402 (2002); Phys.Rev.B, 073406 (2001); Appl.Phys.Lett. 81, 3031 (2002); Appl.Phys.Lett. 77, 2891 (2000)

70. A. Yu. Kasumov, H. Bouchiat, et al., Science 284, 1508 (1999); Phys.Rev.B 68, 214521 (2003)

71. S.Bellucci and P.Onorato, Cond-mat 0504170

72. M. Kociak, H. Bouchiat, et al., Phys. Rev. Lett. 86, 2416 (2001)

73. M.Tinkam, Introduction to Superconductivity (McGraw-Hill, New York 1996)

74. J.Gonzalez, Phys.Rev.Lett. 88, 076403 (2002); A.Sedeki, et al., Phys.Rev.B 65, 140515(R) (2002); J.Gonzalez, Phys.Rev.Lett. 87, 136401 (2001)

75. J.Haruyama, et al., Physica Stat. Sol. (b) 242(2), 265 (2005) 


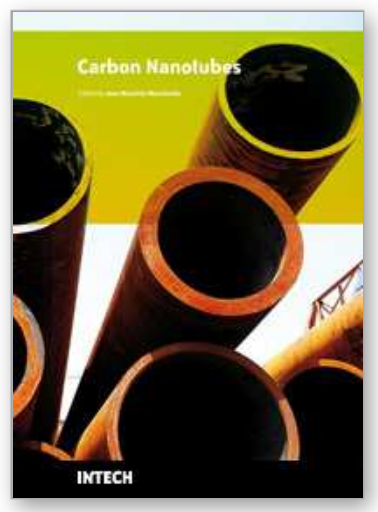

\author{
Carbon Nanotubes \\ Edited by Jose Mauricio Marulanda
}

ISBN 978-953-307-054-4

Hard cover, 766 pages

Publisher InTech

Published online 01, March, 2010

Published in print edition March, 2010

This book has been outlined as follows: A review on the literature and increasing research interests in the field of carbon nanotubes. Fabrication techniques followed by an analysis on the physical properties of carbon nanotubes. The device physics of implemented carbon nanotubes applications along with proposed models in an effort to describe their behavior in circuits and interconnects. And ultimately, the book pursues a significant amount of work in applications of carbon nanotubes in sensors, nanoparticles and nanostructures, and biotechnology. Readers of this book should have a strong background on physical electronics and semiconductor device physics. Philanthropists and readers with strong background in quantum transport physics and semiconductors materials could definitely benefit from the results presented in the chapters of this book. Especially, those with research interests in the areas of nanoparticles and nanotechnology.

\title{
How to reference
}

In order to correctly reference this scholarly work, feel free to copy and paste the following:

Junji Haruyama (2010). Superconductivity in Carbon Nanotubes, Carbon Nanotubes, Jose Mauricio Marulanda (Ed.), ISBN: 978-953-307-054-4, InTech, Available from: http://www.intechopen.com/books/carbonnanotubes/superconductivity-in-carbon-nanotubes

\section{INTECH}

open science | open minds

\section{InTech Europe}

University Campus STeP Ri

Slavka Krautzeka 83/A

51000 Rijeka, Croatia

Phone: +385 (51) 770447

Fax: +385 (51) 686166

www.intechopen.com

\section{InTech China}

Unit 405, Office Block, Hotel Equatorial Shanghai

No.65, Yan An Road (West), Shanghai, 200040, China 中国上海市延安西路65号上海国际贵都大饭店办公楼 405 单元

Phone: +86-21-62489820

Fax: +86-21-62489821 
(C) 2010 The Author(s). Licensee IntechOpen. This chapter is distributed under the terms of the Creative Commons Attribution-NonCommercialShareAlike-3.0 License, which permits use, distribution and reproduction for non-commercial purposes, provided the original is properly cited and derivative works building on this content are distributed under the same license. 